\title{
Article
}

\section{MHC-I peptides get out of the groove and enable a novel mechanism of HIV-1 escape}

Pymm, Phillip, Illing, Patricia T, Ramarathinam, Sri H, O'connor, Geraldine, Hughes, Victoria A, Hitchen, Corinne, Price, David A, Ho, Bosco K, McVicar, Daniel W, Brooks, Andrew G, Purcell, Anthony W, Rossjohn, Jamie and Vivian, Julian P

Available at http://clok.uclan.ac.uk/25533/

Pymm, Phillip, Illing, Patricia T, Ramarathinam, Sri H, O'connor, Geraldine ORCID: 0000-0002-7440-162X, Hughes, Victoria A, Hitchen, Corinne, Price, David A, Ho, Bosco K, McVicar, Daniel W et al (2017) MHC-I peptides get out of the groove and enable a novel mechanism of HIV-1 escape. Nature Structural \& Molecular Biology, 24 (4). pp. 387-394. ISSN 1545-9993

It is advisable to refer to the publisher's version if you intend to cite from the work. http://dx.doi.org/10.1038/nsmb.3381

For more information about UCLan's research in this area go to http://www.uclan.ac.uk/researchgroups/ and search for <name of research Group>.

For information about Research generally at UCLan please go to http://www.uclan.ac.uk/research/

All outputs in CLoK are protected by Intellectual Property Rights law, including Copyright law. Copyright, IPR and Moral Rights for the works on this site are retained by the individual authors and/or other copyright owners. Terms and conditions for use of this material are defined in the policies page. 


\section{MHC-I peptides get out of the groove and enable a novel mechanism of HIV-1 escape}

Phillip Pymm ${ }^{1,2^{*}}$, Patricia Illing ${ }^{1 *}$, Sri Ramarathinam ${ }^{1 *}$, Geraldine O’Connor ${ }^{3}$, Victoria A. Hughes $^{1,2}$, Lucy C. Sullivan ${ }^{3}$, Corinne Hitchen ${ }^{1}$, David A. Price ${ }^{4,5}$, Bosco K. Ho ${ }^{1}$, Daniel W McVicar $^{6}$, Andrew G. Brooks ${ }^{3}$, Anthony W. Purcell ${ }^{\# 1}$, Jamie Rossjohn ${ }^{\# 1,2,4}$ \& Julian Vivian ${ }^{\# 1,2}$

${ }^{1}$ Infection and Immunity Program \& Department of Biochemistry and Molecular Biology, Biomedicine Discovery Institute, Monash University, Clayton, Victoria 3800, Australia

${ }^{2}$ Australian Research Council Centre of Excellence in Advanced Molecular Imaging, Monash University, Clayton, Victoria 3800, Australia

${ }^{3}$ Department of Microbiology and Immunology, Peter Doherty Institute for Infection and Immunity, The University of Melbourne, Parkville, Victoria 3010, Australia

${ }^{4}$ Institute of Infection and Immunity, Cardiff University, School of Medicine, Heath Park, Cardiff, CF14 4XN, UK

${ }^{5}$ Human Immunology Section, Vaccine Research Center, National Institute of Allergy and Infectious Diseases, National Institutes of Health, Bethesda, MD 20892, USA

${ }^{6}$ Cancer and Inflammation Program, National Cancer Institute-Frederick, Frederick, MD 21702, USA

* Joint first authors

\#Joint senior and corresponding authors: Anthony Purcell (anthony.purcell@monash.edu), Jamie Rossjohn (jamie.rossjohn@monash.edu) and Julian Vivian ( julian.vivian@monash.edu) 


\section{Abstract}

Major histocompatibility complex class I (MHC-I) molecules play a crucial role in adaptive and innate immunity by capturing peptides for presentation to T-cells and natural killer (NK) cells, respectively. The termini of these peptides are tethered at opposing ends of the MHC-I molecule, although the extent to which other modes of presentation occur is unknown. Here, we show that $20 \%$ of the human leukocyte antigen (HLA)-B*57:01-restricted peptide repertoire comprises $\mathbf{N}$-terminally extended sets characterised by a common motif at position (P)-1 to P2. Crystal structures of HLA-B*57:01 presenting N-terminally extended peptides, including the immunodominant HIV-1 Gag epitope TW10 (TSTLQEQIGW; residues 240249), revealed that the $\mathrm{N}$-terminus protruded from the peptide-binding groove in a conserved manner. Notably, the common escape mutant TSNLQEQIGW bound HLA-B*57:01 in the canonical fashion and adopted a dramatically different conformation that also impacted recognition by killer immunoglobulin-like receptor (KIR)3DL1. These results define a previously uncharacterised feature of the HLA-I immunopeptidome with important implications for viral immune escape.

\section{Introduction}

Human leukocyte antigen class I (HLA-I) molecules are a vital component of cellular immunity, presenting endogenous peptide epitopes on the cell surface for scrutiny by $\mathrm{CD} 8^{+} \mathrm{T}$-cells and natural killer cells (NK cells). These peptides represent a mix of intracellular proteins that have been proteolytically degraded and during the course of a viral infection will include epitopes originating from the invading pathogen. HLA-I molecules bind compatible peptides via a specialised groove capped by a conserved network of residues that form hydrogen $(\mathrm{H})$ bonds with the $\mathrm{N}$ - and $\mathrm{C}$-termini of the peptide ${ }^{1}$. The peptide is further anchored at specific pockets spaced along the groove, typically at the P2 and P $\Omega$ positions for many HLA allotypes 2, 3, 4, 5, 6. The nature of these 'anchoring pockets' differs between HLA-I molecules thus defining their unique peptide repertoires 6,7 .

The closed-end nature of the HLA-I antigen (Ag)-binding cleft generally constrains the repertoire of peptides to 9-11 amino acids in length. Some longer HLA-I-restricted peptides have been described, but on account of the conserved polar interaction network with the $\mathrm{N}$ - and C-termini in the P1 and P $\quad \square \square \square \square \square \square \square$, longer peptides are canonically accommodated in the groove by adopting a 'bulged' conformation, where the central portion of the peptide epitope projects from the 
groove $8,9,10$. The T-cell receptor (TCR) binds these bulged regions of the peptide whilst simultaneously contacting the HLA-I molecule. Isolated reports have described peptides that protrude at the C-terminus from the peptide-binding groove with the $\mathrm{P} \Omega-1$ residue acting as an alternate C-terminal anchor, although the extent to which this represents a common occurrence remains unclear ${ }^{11,12}$. Such non-canonical HLA-I-peptide landscapes are important because they potentially form unique contact surfaces for TCR and killer immunoglobulin-like receptor (KIR) recognition with attendant implications for disease-relevant immune responses ${ }^{12,13,14}$. In contrast, it is not known whether peptides can protrude from the N-terminus of the HLA-I Ag-binding cleft.

The $H L A-B^{*} 57: 01$ allele is associated with slower HIV-1 disease progression $15,16,17,18,19$ and autoimmune reactions, including hypersensitivity, to the anti-viral drug abacavir ${ }^{20,21,22}$. In HIV-1infected individuals, the expressed HLA-B*57:01 allomorph elicits robust $\mathrm{CD}^{+} \mathrm{T}$-cell responses that are thought to control viremia. Four epitopes in particular within the p24 Gag protein (TW10, IW9, QW9 and KF11) are associated with such protective immunity 16, 23, 24, 25. Cellular immune responses against these epitopes drive the selection of amino acid mutations that allow viral escape from the immune system 26, 27, 28 . A common escape mutation in the p24 Gag epitope TW10 (TSTLQEQIGW; residues 240-249)TW10 is T242N, which lies at position (P)3 of the epitope (T3N) $29,30,31,32$. The emergence of this variant coincides with loss of the original CD8 ${ }^{+} \mathrm{T}$-cell response in many patients ${ }^{25,30}$ and impaired viremic control, especially after the acquisition of compensatory mutations ${ }^{28,33}$. In addition, HLA-B*57:01 is a ligand for members of the KIR3DL1 family, and there is a strong genetic association across the KIR3DL1-HLA-B*57 axis with delayed progression to AIDS $34,35,36$.

Here, we show that the repertoire of endogenous and viral peptides presented by HLA-B*57:01 contains sets of both $\mathrm{N}$ - and C-terminally extended epitopes. A characteristic motif was identified as a signature of N-terminally extended peptides and structural studies revealed a non-canonical binding mode whereby the N-terminal residue overhangs the peptide-binding groove of HLAB*57:01. This novel mode of peptide binding enables HIV-1 to escape immune recognition via a register shift within the TW10 epitope that alters the presented antigenic structure, upon mutation to T3N.

\section{Results}

Structure of HLA-B*57:01-TW10 in complex with KIR3DL1

To extend our previous analysis of KIR3DL1 bound to HLA-B*57:01 presenting a self-peptide, we determined the crystal structure of KIR3DL1*001-HLA-B*57:01 in complex with the HIV-1 p24 
Gag epitope TW10 (TSTLQEQIGW; residues 240-249). Crystals of KIR3DL1-HLA-B*57:01TW10 diffracted to a resolution of $2.0 \AA$ and the final model was refined to $R_{\text {factor }}$ and $R_{\text {free }}$ values of $20.3 \%$ and $23.9 \%$, respectively (Table $\mathbf{1}$ ).

The mode of KIR3DL1 recognition of the viral epitope was essentially identical to that of the selfpeptide bound to HLA-B*57:01 ${ }^{37}$ (Figure 1a). The three KIR3DL1 domains engaged HLAB*57:01 in a conserved orientation with the D0 domain docking at the side and the D1 and D2 domains docking at the C-terminal end of the peptide binding cleft, above the $\alpha 1$ and $\alpha 2$ helices respectively (Figure 1a). The D1 domain interacted with the Bw4 motif on the $\alpha 1$ helix. In the course of our analysis, we noted that the TW10 epitope adopted a unique conformation not previously observed for HLA-I-peptide complexes. Namely, the N-terminal region of the TW10 epitope protruded from the Ag-binding cleft and thus did not occupy the A pocket (Figure 1b). Instead, the second and third residues of the TW10 epitope were located in the A and B pockets, respectively (Figure 1b). Comparison with available HLA-B*57:01-peptide structures 21, 37, 38 showed that, with the exception of a $0.7 \AA$ shift of Trp167 (not shown), there were minimal structural distortions to the peptide-binding cleft that accompanied this previously unobserved peptide conformation (r.m.s.d. $<0.25 \AA$ over C $\alpha$ positions $1-180$ ). Due to the closed nature of the peptide-binding groove, the P-1 residue of the peptide projected at a right-angle from the plane of the floor of the groove, with the side-chain pointing back along the length of the peptide (Figure 1c). Contacts between P-1-Thr and HLA-B*57:01 were limited to water-mediated H-bonds between Trp167 and the P-1-Thr peptide backbone and van Der Waals interactions between Glu63 and Leu163 and the P-1-Thr1 side-chain (Figure 1c). Contacts between the P-1Thr and the rest of the peptide included water-mediated H-bonds with P2-Thr and P4-Gln (Figure 1c).

The A pocket is characterised by a highly conserved set of residues including Tyr7 and Tyr171 which contribute a network of $\mathrm{H}$-bonds that stabilises the $\mathrm{N}$-terminus of the presented peptide. In the HLA-B*57:01-TW10 complex, the P1-Ser residue of the TW10 epitope occupied the A pocket of HLA-B*57:01 and, as such, maintained a network of H-bonds with Tyr7 and Tyr171 (Figure 1d). In so doing, the P1-Ser sidechain was rotated approximately $180^{\circ}$ from the canonical positioning of a P1 anchored residue. Accordingly, the slippage of TW10 from the groove of HLAB*57:01 was accompanied by distortion of the peptide at the P1 residue and not by structural distortion of the peptide-binding groove. As peptide repertoire data for HLA-B*57:01 had previously demonstrated the P2 anchor preference for Ser or $\mathrm{Thr}^{21}$ it was conceivable that either P2-Ser or P3-Thr could act as N-terminal anchors for TW10. However, the high-resolution structure of HLA-B*57:01-TW10 displayed unambiguous electron density across the peptide, thereby 
indicating that only one conformation of TW10 was present in the crystal lattice. Further, it was confirmed by the use of hydrogen-deuterium exchange with mass spectrometry that, compared to the peptide free in solution, TW10 adopts a single conformation whilst in complex with B*57:01 (Figure 2). Thus, the P-1 to $\mathrm{P} 2$ residues enable the peptide to extend from the peptide-binding cleft of HLA-B*57:01.

\section{$N$-terminally extended peptides within the HLA-B*57:01 immunopeptidome}

To determine whether N-terminally extended peptides are a general feature of the HLA-B*57:01 restricted immunopeptidome, we sought to define the range of self-peptides and HIV-Gag epitopes presentated by HLA-B*57:01. Peptides were isolated from C1R cells transfected with expression constructs for HLA-B*57:01 and HIV-1-Gag and sequenced using tandem mass spectrometry. This data set was: (i) filtered for peptides known to bind the endogenous HLA-I and HLA-II of the parental cell line, or bearing the peptide binding motifs of HLA-Cw4 and HLA-B*35:03 (endogenous HLA-I of C1R cells) (14); and (ii) further augmented with previously defined peptides from the immunopeptidome of HLA-B*57:01 (24), which collectively amounted to 11954 peptides (Supplementary Table 1). As previously described, HLA-B*57:01 ligands were predominantly 911 amino acids in length and showed enrichment of S/T/A at the P2 anchor, and aromatic residues at the C-terminus (Figure 3) ${ }^{21}$. In addition, a number of peptides were identified that contained these shorter 9-11 amino acid peptides with an N-terminal extension.

Extended sets of peptides were defined based on the following criteria: i) minimal core sequence of 7-11 amino acids, ii) maximal sequence $>9$ amino acids. 1275 sets of peptides met these criteria, of which $17 \%, 18 \%$ and $12 \%$ of 9,10 and 11 residue peptides fell within these criteria and possessed only $\mathrm{N}$-terminal extensions, whilst far fewer were purely $\mathrm{C}$-terminally extended (3\%, 3\% and $2 \%$ respectively) (Figure 3a). To define the sequence features that were predisposed to Nterminal extensions, peptides exhibiting N-terminal heterogeneity were aligned based on the minimal sequence P1 (Supplementary Table 2). The motif was visualised as a sequence logo encompassing P-1 (1 residue N-terminal of P1) to P3 using Icelogo ${ }^{39}$. A similar motif was also generated from the $\mathrm{P} 1$ to $\mathrm{P} 3$ of all 9-11 residue peptides within the HLA-B*57:01 peptide data set and aligned at P1 for comparison (Figures 3b and 3c). Whilst the global HLA-B*57:01 9-11 amino acid peptide motif shows enrichment of $\mathrm{K} / \mathrm{I} / \mathrm{R}$ at $\mathrm{P} 1$, it was observed that $\mathrm{N}$-terminally extended peptides were enriched for S/T/A at P1 and P-1. Given that S/T/A are preferred P2 anchors for HLA-B*57:01, these residues could potentially be acting as alternate anchor sites in longer peptides, resulting in bulged conformations within the peptide-binding groove. However given the appearance of numerous sets where a minimal core sequence was found with several 
extension lengths (e.g. Set 233: STTSVASSW, TSTTSVASSW, DTSTTSVASSW, HDTSTTSVASSW, SHDTSTTSVASSW, SSHDTSTTSVASSW and TASSHDTSTTSVASSW) not all of which contained S/T/A at the second residue, it seemed likely that the excess residues might overhang the cleft. Thus, we defined the N-terminal protrusion motif as S/T/A at P-1 to P2.

The TW10 epitope is part of a N-terminally extended set of peptides restricted by HLA-B*57:01

Of the 11954 peptides used in this analysis 9 were derived from the Gag polypeptide. Amongst the Gag derived epitopes, two N-terminally extended epitope sets were observed, QW9 and TW10 (Figure 4a, Set 33 and Set 500 Supplementary Table 2). The TW10 epitope was the minimal peptide observed within its $\mathrm{N}$-terminally extended set that contained multiple extension lengths (Figure 4a). To determine the relative abundance of each peptide in the set, we measured the extracted ion chromatogram specific for each of the peptides (Figure 4b). The N-terminally extended AGTTSTLQEQIGW peptide was the most intense ion and by inference the most abundant, followed by TTSTLQEQIGW, GTTSTLQEQIGW and TW10 (Figure 4b). Two longer N-terminally extended peptides were also identified (DIAGTTSTLQEQIGW and SDIAGTTSTLQEQIGW) at significantly lower levels (Figure 4b). Taken together, our data suggest that a common motif characterises N-terminally protruding HLA-B*57:01-restricted peptides regardless of self or viral origin.

Structure of HLA-B*57:01 in complex with $N$-terminally extended self peptides

To further probe the structural nature of the N-terminal extension motif we investigated three epitopes from the self-repertoire of HLA-B*57:01. The selected peptides were TSTTSVASSW (UNP Q14679), TSTFEDVKILAF (UNP Q6YHU6) and SSTRGISQLW (UNP A8CG34) each of which formed part of a nested set (Supplementary Table 2 Set 233, Set 926 and Set 736 respectively). Crystals of the refolded HLA-B*57:01-peptide complexes formed in the space group $P 22_{1} 2_{1}$ with unit cell dimensions of $\sim a=50, b=82$, and $c=110 \AA$, and diffracted to high resolution (Table 1).

All these HLA-B*57:01-peptide complexes adopted the non-canonical conformation observed for HLA-B*57:01-TW10. Namely, the P2-Ser of TSTTSVASSW, TSTFEDVKILAF and SSTRGISQLW occupied the A pocket, which normally accommodates the N-terminal amide. The $\mathrm{H}$-bonding network at the N-terminus of the peptide-binding groove was similarly conserved, with the hydroxyl of the P2-Ser maintaining the contacts seen in canonically bound peptides (Figures 5a-c). Accordingly, the P-1 to $\mathrm{P} 2$ motif identified in the repertoire analysis is a common motif that enables the peptide to protrude from the N-terminus of HLA-B*57:01. 


\section{The structural basis of viral escape via the T3N mutation in TW10}

The TW10 epitope undergoes a common and rapid substitution at the $\mathrm{N}$-terminus, namely T3N (TSNLQEQIGW), to facilitate HIV-1 immune escape in HLA-B*57:01+ individuals. To address this phenomenon at the molecular level, we determined the crystal structure of HLA-B*57:01 in complex with the T3N peptide and KIR3DL1 (Table 1). Overall, the contacts between KIR3DL1 and HLA-B*57:01 were conserved between the TW10 and T3N structures. However, contacts between KIR3DL1 and the HLA-bound peptide were markedly different for TW10 and T3N. KIR3DL1 formed two contacts with the T3N peptide. The D1 Gly116 bound P7-Gln, whilst Tyr200 at the D1/D2 hinge-loop bound P8-Ile (Figure 6a). In contrast, KIR3DL1 did not directly contact the TW10 peptide. Instead, water-mediated contacts were formed between Tyr200 and the backbones of P8-Gly and P9-Trp (Figure 6b). KIR3DL1 therefore interacted differently with TW10 and T3N as a result of changes in the bound peptide conformation (detailed below).

The Thr3Asn substitution caused the peptide to adopt a conventional anchoring mode bound to HLA-B*57:01 with the A and B pockets occupied by P1-Thr and P2-Ser, respectively (Figure 6c). Notably, the HLA-B*57:01-TW10 complex was more stable than the HLA-B*57:01-T3N complex, with a melting temperature of $61.8^{\circ} \mathrm{C}$ compared with $55.0^{\circ} \mathrm{C}$ in circular dichroism assays (Supplementary Figure 1). The inability of Asn3 to occupy the B pocket is consistent with previous HLA-B*57:01 peptide elution data in which Asn was not observed as anchor residue at position $2^{21}$. The change in anchoring at P2 shifted the peptide register across all positions except the C-terminal anchor and P9-Gly (Figure 6d). Accordingly, the secondary anchor positions also differed between TW10 and T3N. Specifically, the TW10 secondary anchors at P3-Leu, P6-Gln and P7-Ile were reassigned to P3-Asn, P5-Gln and P6-Glu, respectively, in T3N. Consequently, the P-1Thr, P4-Gln and P5-Glu side-chains were considerably solvent exposed in TW10, whereas P4-Leu, P5-Gln and P8-Ile were considerably solvent exposed in T3N (Figure 6d). Thus, the Thr3Asn mutation shifts the peptide register within the peptide-binding groove, resulting in the presentation of an altered peptide conformation to immune cell receptors.

\section{Escape mutants in the TW10 epitope abrogate KIR3DL1 recognition}

To determine whether the T3N escape mutation was able to maintain binding to the inhibitory NK cell receptor KIR3DL1, the HLA-B*57:01-TW10 and -T3N complexes were coupled to a CM5 chip with the anti-HLA monoclonal antibody W6/32 and the affinity to KIR3DL1*001 measured by surface plasmon resonance (SPR). The TW10 complex was observed to bind more strongly to KIR3DL1 than T3N, with affinities of $\sim 60 \mu \mathrm{M}$ and $\sim 100 \mu \mathrm{M}$ respectively (Figure 7a). 
To assess the impact of mutation within the TW10 epitope on NK cell function, the binding of T3N, and a second common TW10 escape variant G9D, was measured. Tetramer binding of HLA B*5701-TW10, T3N and G9D to KIR3DL1 transfected HEK293T cells was compared for common KIR3DL1 allotypes within the population $(* 001, * 005$ and *015). Recognition of the HLA B*57:01 tetramer was abrogated for the T3N escape mutation across all KIR3DL1 allotypes tested (Figure 7b). However, the degree of abrogation varied with KIR3DL1 allotype, with KIR3DL1*005 being the least sensitive to the G9D mutation ( $50 \%$ of wild-type binding), and *015 the most sensitive to changes in the epitope (Figure 7b).

The relative contribution of the residues of KIR3DL1*001 to the HLA-B*57:01-TW10 and T3N interfaces was further probed by mutational analysis. Overall, there were distinct differences in the recognition of the HLA-peptide complexes (Figure 7c, d). Looking at these differences by KIR3DL1 domain, it was observed that within the D0 domain, the T3N complex was comparatively less sensitive to substitution at position F9 than TW10, whilst being more sensitive to substitution at F34 (Figure 7c, d). Similarly, clear differences in the recognition by the KIR3DL1 D1 domain were noted. That is, substitution at positions G138 and S140 resulted in significantly different recognition of TW10 yet had limited impact on T3N recognition, whilst the L166A mutation was sufficient to restore T3N binding to wild-type (TW10) levels (Figure 7c, d). Substitutions across the KIR3DL1 D2 domain resulted in comparable contributions from residues Try200, Glu201, Ser277, Ser228, Phe276, His278 and Ser279. Contrary to this, Arg277Ala abrogated T3N binding whilst improving recognition of TW10.

Taken together, these analyses demonstrate that the viral escape mutant T3N abrogates KIR3DL1 recognition, with the degree of abrogation dependent upon KIR3DL1 allotype. Furthermore, the KIR3DL1 residue Leu166, that sat proximal to the interface with the peptide, was central to dictating the reduced affinity for the escape variant. Thus, whilst the altered peptide conformation of $\mathrm{T} 3 \mathrm{~N}$ results in additional direct contacts to KIR3DL1, it nonetheless serves to limit the interaction. Accordingly, whilst the HIV escape mutants within the TW10 epitope evade T-cell recognition, they are likely more vulnerable to NK lysis via KIR3DL1 disinhibition.

\section{Discussion}

HLA-II molecules possess open-ended Ag-binding clefts that allow peptides to extend from the groove at both the $\mathrm{N}$ - and C-termini. In contrast, the $\mathrm{N}$ - and C-terminal pockets of HLA-I molecules are closed, thereby restricting the bound peptides to a typical length of 9-11 amino acids. Nevertheless, longer MHC-I-restricted epitopes have been described that adopt a centrally bulged 
conformation whilst maintaining the $\mathrm{N}$ - and C-terminal anchor residues. In addition, there have been isolated reports of peptides with residues overhanging the C-terminal pocket of HLA-A*02:01 12,40 and $\mathrm{H} 2-\mathrm{M} 3{ }^{41}$. It was not clear, however, whether such overhangs could occur beyond the more buried N-terminal pocket.

Our comprehensive analysis of the repertoire of peptides naturally presented by HLA B*57:01, shows that approximately $27 \%$ are clustered into extended-sets, most of which are solely Nterminally $(20 \%)$ extended, whilst the remaining show either C-terminal extensions or a mix of extension at both $\mathrm{N}$ - and $\mathrm{C}$-termini. We also demonstrate that $\mathrm{N}$-terminally extended peptides are accommodated via protrusion from the groove. From the amino acid enrichment data for Nterminally extended HLA-B*57:01 epitopes and our structure-based insights we can devise rules that predict the P-1 overhang. Namely: (i) The peptide should be a minimum of 1 residue longer than the minimal epitope length that can be presented; (ii) the P2 residue should be a residue that favours binding in the B pocket and (iii) the P1 residue should be smaller than leucine to enable a $180^{\circ}$ rotation within the A pocket. Accordingly, we define a new mechanism that allows MHC-I molecules to present lengthy epitopes.

Viruses have developed numerous strategies to evade HLA-mediated immune detection, including mechanisms that directly interfere with the presentation of viral epitopes and HLA molecules on the cell surface ${ }^{42,43,44}$. For example, HIV-1 can increase cellular endocytosis of HLA molecules via $n f^{45,46}$, limit HLA transcription and peptide processing via tat ${ }^{47,48}$ and suppress TAP-mediated peptide transport into the endoplasmic reticulum ${ }^{49}$. Under selection pressure from the immune system, HIV-1 also mutates rapidly to evade cytotoxic $\mathrm{T}$ lymphocyte responses. These epitopecentric escape mutations typically alter the conformation of exposed residues that interact with the T-cell receptor $50,51,52,53$ or abrogate peptide presentation via effects on antigen processing or HLAI binding ${ }^{54,55}$. Here, we report a novel mode of escape whereby the common T3N mutant exploits the P-1 overhang of TW10 and the anchor residue preferences in the A and B pockets to shift the register of the peptide within HLA-B*57:01. This structural rearrangement alters the conformation of exposed peptide residues to enable immune escape $29,30,31,32$.

There has been significant interest in the idea that KIR-dependent recognition of HLA-I can shape the nature of viral escape mutations, raising the possibility that NK cells may exert some selective pressure on HIV infection ${ }^{56,57}$. However, it is unclear whether the T3N escape variant is able to maintain NK cell inhibition via engagement of KIR3DL1. A previous study using surface plasmon resonance reported a reduced interaction with $\mathrm{T} 3 \mathrm{~N}$ presented by HLA-B*57:03 ${ }^{31}$. In contrast, 
another study based on tetramer staining of KIR3DL1-transfected cell lines reported no interaction 58. Although this discrepancy likely reflects the different experimental approaches, it is clear from our structural, SPR and tetramer staining data that KIR3DL1 recognises T3N with a reduced affinity compared to that of the TW10 epitope bound to HLA-B*57:01. Notably, the degree to which binding was reduced was observed in a KIR3DL1-allotype dependent manner. There are also well-described C-terminal escape mutations within the TW10 epitope, of which G9D has similarly been shown to abrogate KIR3DL1 recognition in a KIR3DL1-allotype dependent manner ${ }^{59}$. This is in-line with recent reports on KIR3DL1 allomorph specificity ${ }^{60}$ and suggests that KIR3DL1 polymorphism may be an important determinant shaping viral fitness. Accordingly, there appears to be a molecular tension between the innate and adaptive arms of the immune system as they converge to target a common HIV-I determinant presented by HLA-B*57:01. In sum, our structural and proteomics analyses reveal a novel mechanism of viral immune escape, whereby HIV-1 mutates to exploit a previously undescribed mode of peptide presentation by MHC-I.

Methods

\section{Isolation of MHCI peptide ligands}

C1R transfectants were grown to high density in RPMI 1640 (Thermo Fisher Scientific, Waltham, MA) supplemented with $10 \%$ fetal calf serum (FCS; Bovogen Biologicals Pty. Ltd., Melbourne, Australia), 7.5 mM HEPES (MP Biomedicals, Eschwege, Germany), $150 \mu \mathrm{g} / \mathrm{mL}$ streptomycin (Sigma-Aldrich, St. Louis, MO), 150 U/mL benzylpenicillin (CSL, Parkville, Australia), 2 mM Lglutamine (MP Biomedicals), $76 \mu \mathrm{M} \beta$-mercaptoethylamine (Sigma-Aldrich) and $150 \mu \mathrm{M}$ nonessential amino acids (Life Technologies, Carlsbad, CA). Transfectants were maintained under hygromycin $(0.3 \mathrm{mg} / \mathrm{mL})$ and $\mathrm{G} 418(0.5 \mathrm{mg} / \mathrm{mL})$ selection. Cells were harvested by centrifugation $\left(1200 \mathrm{~g}, 20 \mathrm{~min}, 4^{\circ} \mathrm{C}\right)$ and snap frozen in liquid nitrogen. Clarified lysates were generated from $5 \mathrm{x}$ $10^{9}$ cells using a combination of cryogenic milling and detergent-based lysis. HLA-peptide complexes were immunoaffinity-purified from cell lysates using the W6/32 monoclonal antibody in solid phase as described previously ${ }^{61,62}$. Bound complexes were eluted by acidification with $10 \%$ acetic acid and fractionated using a $4.6 \mathrm{~mm}$ internal diameter x $100 \mathrm{~mm}$ long monolithic reversedphase (RP) C18 high performance liquid chromatography (HPLC) column (Chromolith SpeedROD; Merck Millipore, Darmstadt, Germany) with the ÄKTAmicro ${ }^{\mathrm{TM}}$ HPLC system (GE Healthcare, Little Chalfont, UK). The mobile phase consisted of buffer A (0.1\% trifluoroacetic acid; Thermo Fisher Scientific) and buffer B (80\% acetonitrile, $0.1 \%$ trifluoroacetic acid; Thermo Fisher Scientific). HLA-peptide mixtures were loaded onto the column at a flow rate of $1 \mathrm{~mL} / \mathrm{min}$ with separation based on a B gradient of $2-40 \%$ for $4 \mathrm{~min}, 40-45 \%$ for another $4 \mathrm{~min}$ and a final rapid 2 min increase to $100 \%$. Fractions $(500 \mu \mathrm{L})$ were collected, vacuum-concentrated to $10 \mu \mathrm{L}$ and 
diluted in $0.1 \%$ formic acid to reduce the acetonitrile concentration. The Gag plasmid was a kind gift from Johnson Mak (Deakin University, Melbourne Australia).

Liquid chromatography-tandem mass spectrometry (LC-MS/MS) sequencing of MHC-I-bound peptides

For LC-MS/MS acquisition, peptide-containing fractions were loaded onto a microfluidic trap column packed with ChromXP C18-CL $3 \mu \mathrm{m}$ particles (300 $\AA$ nominal pore size; equilibrated in $0.1 \%$ formic acid/ $2 \%$ acetonitrile) at $5 \mu \mathrm{l} / \mathrm{min}$ using a NanoUltra cHiPLC system (Eksigent). An

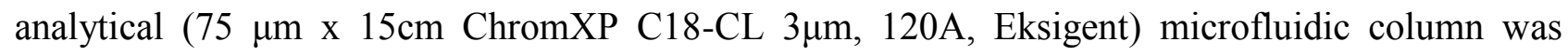
switched in line and peptides separated using linear gradient elution 0-30\% buffer B (80\% acetonitrile, $0.1 \%$ formic acid) over $50 \mathrm{~min}$ and 30-80\% over $5 \mathrm{~min}$ flowing at $300 \mathrm{~nL} / \mathrm{min}$. Separated peptides were analysed using an AB SCIEX 5600+ TripleTOF mass spectrometer equipped with a Nanospray III ion source and accumulating up to $20 \mathrm{MS} / \mathrm{MS}$ spectra per second. The following instrument parameters were used: ion spray voltage (ISVF) was set at $2400 \mathrm{~V}$, curtain gas (CUR) at $25 \mathrm{~L} / \mathrm{min}$, ion source gas(GS1) at $10 \mathrm{~L} / \mathrm{min}$ and an interface heater temperature (IHT) setting of $150^{\circ} \mathrm{C}$. MS/MS switch criteria included ions of $\mathrm{m} / \mathrm{z}>200$ amu, charge state +2 to +5 , intensity $>40 \mathrm{cps}$ and the top 20 ions meeting this criterion were selected for MS/MS per cycle. The instrument was calibrated every four LC runs using [Glu1]-Fibrinopeptide B standard as per manufacturer's instructions.

LC-MS/MS data was searched against the human proteome (UniProt/SwissProt v2014_10) and the HIV NL4-3-gag and AD8-env sequence using ProteinPilot ${ }^{\mathrm{TM}}$ software (version 4.5, SCIEX). A 5\% FDR cut-off was applied. Peptides known to bind the endogenous MHCI of C1R cells (HLA-C*04:01 and HLA-B*35:03) ${ }^{14}$ and those identified as binders of MHCII in similar MHCII isolations in the laboratory and commonly observed contaminants were removed from the data set prior to subsequent analysis of HLA-B*57:01 peptide ligands.

\section{Protein expression and purification}

The HLA-B*57:01 and 32-microglobulin genes were sub-cloned into the pET-30 expression vector and expressed separately into inclusion bodies in E. coli prior to refolding and purification as described previously ${ }^{63}$. Briefly, HLA-B*57:01 was refolded by rapid dilution in a solution containing $100 \mathrm{mM}$ Tris- $\mathrm{HCl} \mathrm{pH}$ 8.0, $400 \mathrm{mM}$ L-arginine- $\mathrm{HCl}, 5 \mathrm{mM}$ reduced glutathione, and 0.5 $\mathrm{mM}$ oxidized glutathione in the presence of $\beta 2$-microglobulin the appropriate peptide. The refolded HLA-B*57:01 complex was purified by anion exchange and size exclusion chromatography. 
KIR3DL1*001 (residues 1 - 299) was sub-cloned into the pHLSec mammalian expression vector with N-terminal 6xHis and secretion tags. KIR3DL1 was expressed and secreted from transiently transfected HEK 293S cells and harvested from the culture media after three by nickel affinity and gel filtration chromatography using an S200 16/60 column (GE Healthcare) in $10 \mathrm{mM}$ Tris pH 8.0, $300 \mathrm{mM} \mathrm{NaCl}$. Purified KIR3DL1 was then concentrated to $15 \mathrm{mg} / \mathrm{mL}$ and deglycosylated with endoglycosidase H (New England Biolabs, Ipswich, MA). The extent of deglycosylation was monitored by SDS-PAGE prior to crystallization trials. For surface plasmon resonance studies, a similar construct of KIR3DL1*001 was prepared in the pFastBac vector and expressed from Hi-5 insect cells (Invitrogen, Carlsbad, CA). KIR3DL1 was purified as above excluding the endoglycosidase $\mathrm{H}$ deglycosylation step.

Hydrogen/deuterium Exchange and analysis by LC-MS.

A sample containing $5 \mu \mathrm{g}(0.1 \mathrm{nmol})$ of peptide or protein complex containing peptide was diluted 24-fold with $50 \mathrm{mM}$ Tris and $50 \mathrm{mM} \mathrm{NaCl}$ dissolved in $\mathrm{D}_{2} \mathrm{O}$ (Cambridge Isotope Laboratories) at $25^{\circ} \mathrm{C}$ to label the sample. The deuteration reaction was quenched at 10 seconds by adding an equal volume of $100 \mathrm{mM} \mathrm{NaH} \mathrm{PO}_{4}(\mathrm{pH} 2.4)$ and quickly frozen in a dry ice-ethanol bath. The frozen sample was quickly thawed and immediately injected onto a micropeptide trap column connected to a C18 HPLC column coupled to a Bruker Micro quadrupole time of flight mass spectrometer. The HLA-bound peptide was separated using a 12 min gradient of $10-45 \%$ acetonitrile at a flow rate of $50 \mu \mathrm{L} / \mathrm{min}$. The micropeptide trap and C18 HPLC column were immersed in ice to minimize back exchange. Because the mass of a peptide increases by one for every amide hydrogen atom exchanged with deuterium, the amount of deuterium can be determined by comparing the mass of the labelled peptide with the mass of the same peptide without the label. The centroid mass of each peptide was determined using the software package MagTran ${ }^{64}$.

\section{Crystallisation, data collection, structure determination and refinement}

The peptide sequences crystallised in complex with HLA-B*57:01 are summarized in Table 2. HLA-B*57:01 binary and ternary complexes with KIR3DL1*001 were concentrated to $\sim 10 \mathrm{mg} / \mathrm{mL}$ and crystallized at $294 \mathrm{~K}$ by the hanging-drop vapour-diffusion method. Binary complexes (TW10, TST-SW10, AST, TSA and YW10) crystallised from a solution comprising $12-20 \%$ PEG 4000, $0.2 \mathrm{M}$ ammonium acetate and $0.1 \mathrm{M}$ tri-sodium citrate $\mathrm{pH} 5.4-5.6$. Ternary complexes (T3N, SW10 and TAT) crystallised from a solution comprising 16\% PEG 3350, 2\% tacsimate pH 5.0 and $0.1 \mathrm{M}$ tri-sodium citrate $\mathrm{pH}$ 5.6. Prior to data collection, crystals were equilibrated in reservoir solution with $10 \%$ glycerol added as a cryoprotectant and then flash-cooled in a stream of liquid nitrogen at $100 \mathrm{~K}$. Data sets were collected at the MX2 beamline (Australian Synchrotron, Victoria). 
The data were recorded on a Quantum-315 CCD detector and were integrated and scaled using MOSFLM and SCALA from the CCP4 program suite ${ }^{65,66,67}$. Details of the data processing statistics are summarised in Table 1. The crystal structures were solved by molecular replacement, as implemented in PHASER ${ }^{68}$ with HLA-B*57:01-LF9 used as the search model (Protein Data Bank accession number: 2RFX). Refinement of the models proceeded with iterative rounds of manual building in COOT ${ }^{69}$ and refinement in PHENIX ${ }^{70}$. The structures were validated with MOLPROBITY ${ }^{71}$. Refinement statistics are summarised in Table 1. Coordinates and structure factors were deposited in the Protein Data Bank under accession numbers XXXX.

\section{Surface Plasmon Resonance}

Surface Plasmon Resonance (SPR) experiments were conducted at $298 \mathrm{~K}$ on a Biacore 3000 instrument using HBS buffer (10 mM HEPES-HCl (pH 7.4), $150 \mathrm{mM} \mathrm{NaCl}$, and $0.005 \%$ surfactant P20 supplied by GE Healthcare). The HLA class I-specific antibody W6/32 was immobilized on a CM5 chip via amine coupling. The pHLA complexes were captured by W6/32 creating a surface density of approximately 700 response units (RU). Concentrations of KIR3DL1*001 (2.37 to $300 \mu \mathrm{M}$ ) were injected over the captured pHLA at $5 \mu \mathrm{l} / \mathrm{min}$. The final response was calculated by subtracting the response of W6/32 alone from the KIR3DL1*001-pHLA-B*5701 complex. The equilibrium data were analyzed using GraphPad Prism. The data are representative of two independent experiments with error bars representing standard error.

\section{Transfection studies}

FLAG tagged KIR3DL1*001 was cloned into the pEF6 vector. Specific nucleotide residues were mutated using the QuikChange II Site Directed Mutagenesis Kit (Stratagene) according to the manufacturer's instructions. These constructs were introduced into HEK293T cells using FuGene ${ }^{\circledR}$ 6 transfection reagent (Roche) according to the manufacturer's instructions. After 48 hours, the cells were harvested and stained with anti-FLAG (clone M2, Sigma Aldrich) antibody or with tetramer for 30 minutes at $4{ }^{\circ} \mathrm{C}$. The cells were then washed and analysed on a Fortessa flow cytometer (BD Biosciences). Analysis of cell surface expression as assessed by staining with anti-FLAG mAb showed that the introduction of the mutations had no substantial effect on expression (data not shown). All transfection data are representative of two independent experiments.

\section{ACKNOWLEDGEMENTS}

This work was supported by project grants from the National Health and Medical Research Council of Australia (NH\&MRC, APP1063829) and Australian Research Council (ARC, DP150104503). A.W.P. is a NH\&MRC Senior Research Fellow. P.T.I. is a NH\&MRC Early Career Fellow. S.H.R. 
is the recipient of an Australian Postgraduate Award. D.A.P. is supported by a Wellcome Trust Senior Investigator Award. J.R. is supported by an ARC Laureate Fellowship. This research was undertaken in part on the MX2 beamline at the Australian Synchrotron, Victoria, Australia.

\section{REFERENCES}

1. Saper MA, B.P., Wiley DC. Refined structure of the human histocompatibility antigen HLA-A2 at 2.6 A resolution. Journal of Molecular Biology 219, 277-319 (1991).

2. Rammensee HG, F.T., Stevanoviíc S. MHC ligands and peptide motifs-first listing. Immunogenetics 41, 178-228 (1995).

3. Deres K, B.W., Faath S, Jung G, Rammensee HG. MHC:peptide binding studies indicate hierarchy of anchor residues.pdf. Cellular Immunology 151, 158-167 (1993).

4. Rammensee HG, F.K., Rötzschke O. Peptides Naturally Presented by MHC Class I Molecules. Annual Review of Immunology 11, 213-244 (1993).

5. Wilson IA, F.D. Structural analysis of MHC class I molecules with bound peptide antigens. Seminars in Immunology 5, 75-80 (1993).

6. Garrett TP, S.M., Bjorkman PJ, Strominger JL, Wiley DC. Specificity pockets for the side chains of peptide antigens in HLA-Aw68. Nature 342, 692-696 (1989).

7. Falk K, R.O., Stevanović S, Jung G, Rammensee HG. Allele-specific motifs revealed by sequencing of self-peptides eluted from MHC molecules. Nature 351, 290-296 (1991).

8. Speir JA, S.J., Joly E, Butcher GW, Wilson IA. Two Different, Highly Exposed, Bulged Structures for an Unusually Long Peptide Bound to Rat MHC Class I RT1-A. Immunity 14, 81-92 (2001).

9. Guo HC, J.T., Garrett TP, Lane WS, Strominger JL, Wiley DC. Different length peptides bind to HLA-Aw68 similarly at their ends but bulge out in the middle. Nature 360, 364366 (1992).

10. Tynan, F.E. et al. High resolution structures of highly bulged viral epitopes bound to major histocompatibility complex class I. Implications for T-cell receptor engagement and T-cell immunodominance. The Journal of biological chemistry 280, 23900-23909 (2005).

11. Collins EJ, G.D., Wiley DC. Three-dimensional structure of a peptide extending from one end of a class I MHC binding site. Nature 371, 626-629 (1994).

12. Tenzer, S. et al. Antigen processing influences HIV-specific cytotoxic Tymphocyte immunodominance. Nature immunology 10, 636-646 (2009). 
13. Anette Stryhn, L.Ø.P., Arne Holm and Søren Buus. Longer peptide can be accommodated in the MHC class I binding site by a protrusion mechanism. Eur J Immunol 30, 3089-3099 (2000).

14. Schittenhelm, R.B., Dudek, N.L., Croft, N.P., Ramarathinam, S.H. \& Purcell, A.W. A comprehensive analysis of constitutive naturally processed and presented HLAC*04:01 (Cw4)-specific peptides. Tissue antigens 83, 174-179 (2014).

15. Carrington, M. \& O'Brien, S.J. The influence of HLA genotype on AIDS. Annual review of medicine 54, 535-551 (2003).

16. Migueles, S.A. et al. HLA B*5701 is highly associated with restriction of virus replication in a subgroup of HIV-infected long term nonprogressors. Proceedings of the National Academy of Sciences of the United States of America 97, 2709-2714 (2000).

17. Gao, X. et al. AIDS restriction HLA allotypes target distinct intervals of HIV-1 pathogenesis. Nature medicine 11, 1290-1292 (2005).

18. Kaslow RA, C.M., Apple R, Park L, Muñoz A, Saah AJ, Goedert JJ, Winkler C, O'Brien SJ, Rinaldo C, Detels R, Blattner W, Phair J, Erlich H, Mann DL. Influence of combinations of human major histocompatibility complex genes on the course of HIV-1 infection. Nature medicine 2, 405-411 (1996).

19. Altfeld, M. et al. Influence of HLA-B57 on clinical presentation and viral control during acute HIV-1 infection. AIDS 17, 2581-2591 (2003).

20. Mallal, S. et al. Association between presence of HLA-B*5701, HLA-DR7, and HLA-DQ3 and hypersensitivity to HIV-1 reverse-transcriptase inhibitor abacavir. The Lancet 359, 727-732 (2002).

21. Illing, P.T. et al. Immune self-reactivity triggered by drug-modified HLA-peptide repertoire. Nature 486, 554-558 (2012).

22. Hetherington, S. et al. Genetic variations in HLA-B region and hypersensitivity reactions to abacavir. The Lancet 359, 1121-1122 (2002).

23. Goulder PJ, B.M., Krausa P, McIntyre K, Crowley S, Morgan B, Edwards A, Giangrande P, Phillips RE, McMichael AJ. Novel, Cross-Restricted, Conserved, and Immunodominant Cytotoxic T Lymphocyte Epitopes in Slow Progressors in HIV Type 1 Infection. AIDS Res Hum Retroviruses 12, 1691-1698 (1996).

24. Klein MR, v.d.B.S., Hovenkamp E, Holwerda AM, Drijfhout JW, Melief CJ, Miedema F. Characterization of HLA-B57-restricted human immunodeficiency virus type 1 Gagand RT-specific cytotoxic T lymphocyte responses. The Journal of General Virology 79, 2191-2201 (1998).

25. Bailey, J.R., Williams, T.M., Siliciano, R.F. \& Blankson, J.N. Maintenance of viral suppression in HIV-1-infected HLA-B*57+ elite suppressors despite CTL escape mutations. J Exp Med 203, 1357-1369 (2006). 
26. Bernardin, F., Kong, D., Peddada, L., Baxter-Lowe, L.A. \& Delwart, E. Human immunodeficiency virus mutations during the first month of infection are preferentially found in known cytotoxic T-lymphocyte epitopes. Journal of virology 79, 11523-11528 (2005).

27. Ganusov, V.V. et al. Fitness costs and diversity of the cytotoxic T lymphocyte (CTL) response determine the rate of CTL escape during acute and chronic phases of HIV infection. Journal of virology 85, 10518-10528 (2011).

28. Martinez-Picado, J. et al. Fitness cost of escape mutations in p24 Gag in association with control of human immunodeficiency virus type 1. Journal of virology $\mathbf{8 0}, 3617-$ 3623 (2006).

29. Novitsky, V. et al. Dynamics and timing of in vivo mutations at Gag residue 242 during primary HIV-1 subtype C infection. Virology 403, 37-46 (2010).

30. Miura, T. et al. HLA-B57/B*5801 human immunodeficiency virus type 1 elite controllers select for rare gag variants associated with reduced viral replication capacity and strong cytotoxic T-lymphocyte [corrected] recognition. Journal of virology 83, 2743-2755 (2009).

31. Brackenridge, S. et al. An early HIV mutation within an HLA-B*57-restricted T cell epitope abrogates binding to the killer inhibitory receptor 3DL1. Journal of virology $\mathbf{8 5}$, 5415-5422 (2011).

32. Crawford, H. et al. Evolution of HLA-B*5703 HIV-1 escape mutations in HLA-B*5703positive individuals and their transmission recipients. J Exp Med 206, 909-921 (2009).

33. Crawford, H. et al. Compensatory mutation partially restores fitness and delays reversion of escape mutation within the immunodominant HLA-B*5703-restricted Gag epitope in chronic human immunodeficiency virus type 1 infection. Journal of virology 81, 8346-8351 (2007).

34. Alter, G. et al. Differential natural killer cell-mediated inhibition of HIV-1 replication based on distinct KIR/HLA subtypes. The Journal of experimental medicine 204, 30273036 (2007).

35. Martin, M.P. et al. Epistatic interaction between KIR3DS1 and HLA-B delays the progression to AIDS. Nat Genet 31, 429-434 (2002).

36. Qi, Y. et al. KIR/HLA pleiotropism: protection against both HIV and opportunistic infections. PLoS Pathog 2, e79 (2006).

37. Vivian, J.P. et al. Killer cell immunoglobulin-like receptor 3DL1-mediated recognition of human leukocyte antigen B. Nature 479, 401-405 (2011).

38. Chessman, D. et al. Human leukocyte antigen class I-restricted activation of CD8+ T cells provides the immunogenetic basis of a systemic drug hypersensitivity. Immunity 28, 822-832 (2008). 
39. Colaert, N., Helsens, K., Martens, L., Vandekerckhove, J. \& Gevaert, K. Improved visualization of protein consensus sequences by iceLogo. Nat Methods 6, 786-787 (2009).

40. Collins, E.J., Garboczi, D.N. \& Wiley, D.C. Three-dimensional structure of a peptide extending from one end of a class I MHC binding site. Nature 371, 626-629 (1994).

41. Wang, C.R. et al. Nonclassical binding of formylated peptide in crystal structure of the MHC class Ib molecule H2-M3. Cell 82, 655-664 (1995).

42. Petersen, J.L., Morris, C.R. \& Solheim, J.C. Virus evasion of MHC class I molecule presentation. Journal of immunology 171, 4473-4478 (2003).

43. Walker, B. \& McMichael, A. The T-cell response to HIV. Cold Spring Harbor perspectives in medicine 2 (2012).

44. Jost, S. \& Altfeld, M. Evasion from NK cell-mediated immune responses by HIV-1. Microbes and infection / Institut Pasteur 14, 904-915 (2012).

45. Greenberg, M.E., Iafrate, A.J. \& Skowronski, J. The SH3 domain-binding surface and an acidic motif in HIV-1 Nef regulate trafficking of class I MHC complexes. The EMBO journal 17, 2777-2789 (1998).

46. Schwartz, O., Marechal, V., Le Gall, S., Lemonnier, F. \& Heard, J.M. Endocytosis of major histocompatibility complex class I molecules is induced by the HIV-1 Nef protein. Nature medicine 2, 338-342 (1996).

47. Howcroft, T.K., Strebel, K., Martin, M.A. \& Singer, D.S. Repression of MHC class I gene promoter activity by two-exon Tat of HIV. Science 260, 1320-1322 (1993).

48. Seeger, M., Ferrell, K., Frank, R. \& Dubiel, W. HIV-1 tat inhibits the 20 S proteasome and its $11 \mathrm{~S}$ regulator-mediated activation. The Journal of biological chemistry 272, 81458148 (1997).

49. Kutsch, O., Vey, T., Kerkau, T., Hunig, T. \& Schimpl, A. HIV type 1 abrogates TAPmediated transport of antigenic peptides presented by MHC class I. Transporter associated with antigen presentation. AIDS Res Hum Retroviruses 18, 1319-1325 (2002).

50. Iglesias, M.C. et al. Escape from highly effective public CD8+ T-cell clonotypes by HIV. Blood 118, 2138-2149 (2011).

51. Liu, Y.C. et al. A molecular basis for the interplay between T cells, viral mutants and human leukocyte antigen micropolymorphism. The Journal of biological chemistry (2014).

52. Shimizu, A. et al. Structure of TCR and antigen complexes at an immunodominant CTL epitope in HIV-1 infection. Scientific reports 3, 3097 (2013).

53. Ladell, K. et al. A molecular basis for the control of preimmune escape variants by HIVspecific CD8+ T cells. Immunity 38, 425-436 (2013). 
54. Goulder, P.J. et al. Evolution and transmission of stable CTL escape mutations in HIV infection. Nature 412, 334-338 (2001).

55. Schneidewind, A. et al. Escape from the dominant HLA-B27-restricted cytotoxic Tlymphocyte response in Gag is associated with a dramatic reduction in human immunodeficiency virus type 1 replication. Journal of virology 81, 12382-12393 (2007).

56. Alter, G. \& Altfeld, M. NK cells in HIV-1 infection: evidence for their role in the control of HIV-1 infection. J Intern Med 265, 29-42 (2009).

57. Lichterfeld, M. et al. A viral CTL escape mutation leading to immunoglobulin-like transcript 4-mediated functional inhibition of myelomonocytic cells. The Journal of experimental medicine 204, 2813-2824 (2007).

58. Fadda, L. et al. Common HIV-1 peptide variants mediate differential binding of KIR3DL1 to HLA-Bw4 molecules. Journal of virology 85, 5970-5974 (2011).

59. O'Connor, G.M. et al. Mutational and structural analysis of KIR3DL1 reveals a lineagedefining allotypic dimorphism that impacts both HLA and peptide sensitivity. Journal of immunology 192, 2875-2884 (2014).

60. Saunders, P.M. et al. Killer cell immunoglobulin-like receptor 3DL1 polymorphism defines distinct hierarchies of HLA class I recognition. The Journal of experimental medicine 213, 791-807 (2016).

61. Dudek, N.L. et al. Constitutive and inflammatory immunopeptidome of pancreatic betacells. Diabetes 61, 3018-3025 (2012).

62. Purcell, A.W. \& Gorman, J.J. The use of post-source decay in matrix-assisted laser desorption/ionisation mass spectrometry to delineate T cell determinants. J Immunol Methods 249, 17-31 (2001).

63. Clements, C.S. et al. The production, purification and crystallization of a soluble heterodimeric form of a highly selected T-cell receptor in its unliganded and liganded state. Acta crystallographica 58, 2131-2134 (2002).

64. Zhang, Z. \& Marshall, A.G. A universal algorithm for fast and automated charge state deconvolution of electrospray mass-to-charge ratio spectra. J Am Soc Mass Spectrom 9, 225-233 (1998).

65. Collaborative. The CCP4 suite: programs for protein crystallography. Acta Crystallographica Section D 50, 760-763 (1994).

66. Evans, P. Scaling and assessment of data quality. Acta crystallographica 62, 72-82 (2006).

67. Leslie, A.G.W. Recent changes to the MOSFLM package for processing film and image plate data. Joint CCP4 + ESF-EAMCB Newsletter on Protein Crystallography 26 (1992). 
68. McCoy, A.J. et al. Phaser crystallographic software. Journal of applied crystallography 40, 658-674 (2007).

69. Emsley, P. \& Cowtan, K. Coot: model-building tools for molecular graphics. Acta crystallographica. Section D, Biological crystallography 60, 2126-2132 (2004).

70. Adams, P.D. et al. PHENIX: a comprehensive Python-based system for macromolecular structure solution. Acta crystallographica. Section D, Biological crystallography 66, 213221 (2010).

71. Chen, V.B. et al. MolProbity: all-atom structure validation for macromolecular crystallography. Acta crystallographica. Section D, Biological crystallography 66, 12-21 (2010). 


\section{FIGURE LEGENDS:}

Figure 1: HLA-B*57:01 in complex with the TW10 peptide. (a) The overall structure of KIR3DL1 in complex with HLA-B*57:01-TW10. (b) Cartoon representation of the crystal structure of HLA B*57:01 (light grey) complexed with the TW10 peptide-TSTLQEQIGW (pink) shown against the $\alpha 1$ helix of the HLA and oriented N-C terminal from left. (c). Orientation of the protruding Thr residue at P-1 and (d) The conserved hydrogen bonding network at the N-terminal end of the HLA B*57:01 is maintained to Ser at P1. Hydrogen bonds are displayed as blue dashed lines.

Figure 2: Hydrogen/deuterium exchange for the TW10 peptide in complex with HLA-B*57:01 and free in solution. (a) Peptide in complex with HLA-B*57:01 at 0 seconds showing the normal isotopic distribution for a singly charged peptide (b) The peptide in complex with HLA-B*57:01 after 10 seconds incubation in $\mathrm{D}_{2} \mathrm{O}$ showing a single Gaussian distribution indicative of a single bound conformation and (c) the peptide free in a solution of $\mathrm{D}_{2} \mathrm{O}$ for 10 seconds showing a bimodal distribution suggesting multiple conformations in solution.

Figure 3: (a) Length distribution of HLA-B*57:01 ligands, showing those classified as part of nested sets. Peptides within the nested sets are then further broken down into the following categories: i. peptides that have been identified as having versions that are extended at the $\mathrm{N}$ terminus, but do not have C-terminally extended versions (blue, $\mathrm{N}$ terminally extended), ii. peptides that have been identified as having versions that are extended at the $\mathrm{C}$ terminus, but do not have Nterminally extended versions (red, $\mathrm{C}$ terminally extended), iii. peptides that have been identified as having versions that are extended at the $\mathrm{N}$ terminus, but also have $\mathrm{C}$ terminally extended versions (green, $\mathrm{N}$ or $\mathrm{C}$ terminally extended), iv. peptides that have been identified with versions that are extended at both the $\mathrm{N}$ and $\mathrm{C}$ terminus but not at either terminus alone (purple, $\mathrm{N}$ and $\mathrm{C}$ terminally extended), and v. peptides that are the maximal sequence of an extended set. Peptides that were not classified as part of an extended set are shown in orange, however it should be noted that extended sets were defined as having a minimal sequence of $<12$ amino acids to minimise ambiguity during motif analysis, thus peptides of 12 amino acids or greater that are not part of extended sets as defined but have extended versions exist within this category. Percentage values show the percentage of 9,10 and 11 amino acid peptides that are part of extended sets and are extended at the $\mathrm{N}$ terminus alone. (b) and (c) Sequence logos showing the percentage difference in abundance of amino acids at each location in the N-terminal portion of HLA-B*57:01 ligands in comparison to their abundance in the human proteome. Logos were generated from the N-terminal portion of all 911 residue peptides in the HLA-B*57:01 data set $(n=8268)$, aligned based on first residue assignment of P1 (b) and from the N-terminal portion of the maximal sequences of nested sets 
containing purely $\mathrm{N}$-terminal extensions, aligned based on the minimal sequences of the nested set and possessing $\mathrm{C}$-terminal aromatic anchors $(\mathrm{n}=972)$ (c). Sequence logos were generated using the iceLogo stand alone version ${ }^{39}$.

Figure 4: The HIV-1 Gag repertoire of HLA-B*57:01. (a) HIV-1 Gag epitopes presented by HLAB*57:01. The QW9 and TW10 epitopes form part of N-terminally extended sets. (b) Relative levels of N-terminally extended variants compared to the HIV-Gag TW10 epitope. Error bars represent mean with $\mathrm{SD} ; \mathrm{n}=3$.

Figure 5: Cartoon representation of the crystal structure of HLA-B*57:01 (light grey) complexed with the TSTFEDVKILAF peptide (blue). (a) HLA-B*57:01 complexed with the TSTFEDVKILAF peptide shown against the $\alpha 1$ helix of the HLA and oriented N-C terminal from left. (b) The network of direct and water-mediated hydrogen bonds (dark blue dashed lines) around the protruding P-1 residue showing the interaction of P-1-Thr with Trp167 and Asn66 of the HLA. (c) The network of conserved hydrogen bonds at the N-terminus of the HLA-B*57:01 peptide-binding groove showing the P1-Ser1 side-chain replacing contacts normally mediated by the N-terminus of the peptide.

Figure 6: Comparison of the TW10 and T3N ternary complex structures with KIR3DL1*001 (a) The interactions between KIR3DL1 (teal) and the T3N peptide (blue). (b) The interactions between KIR3DL1 (teal) and the TW10 peptide (magenta). (c) Cartoon representation of the crystal structure of HLA-B*57:01 (light grey) complexed with the T3N peptide-TSNLQEQIGW (dark blue) shown against the $\alpha 1$ helix of the HLA and oriented N-C terminal from left. Hydrogen bonds are displayed as blue dashed lines. (d) Overlay of the TW10 (pink) and T3N (dark blue) peptide conformations.

Figure 7: HLA B*57:01 TW10 and T3N binding to KIR3DL1 (a) SPR based affinity measurements of the interaction between KIR3DL1*001 and (i) B57:01 TW10 and (ii) B*57:01 T3N complexes (b) Staining of KIR3DL1 allotypes with HLA B*57:01 TW10 (blue) and T3N (grey) tetramers $(0.2 \mu \mathrm{g}$ each), normalised to TW10 binding (c) HLA B*57:01 TW10 (blue) and T3N (grey) tetramer staining of HEK293 cells transfected with KIR3DL1*001 and a panel of KIR3DL1*001 interface residue mutants, normalised to TW10 tetramer binding to KIR3DL1*001 or (d) to the respective tetramer binding of KIR3DL1*001 transfectants. 
Supplementary Figure 1: Circular dichroism readings taken at 222nm over a temperature range of 20-90 ${ }^{\circ} \mathrm{C}$ for the HLA-B*57:01-TW10 complex (a) and the HLA-B*57:01-T3N complex (b). $\mathrm{T}_{\mathrm{m}}$ was calculated by fitting a sigmoidal dose-response curve and taking the IC50 value of the curve.

Supplementary Table 1: List of all peptides isolated from HLA-B*57:01 and their source proteins. Peptides contained in nested sets are indicated. Peptides considered unlikely to be true HLA-B*57:01 ligands due to lack of conformation to consensus motif are noted.

Supplementary Table 2: 1000 purely N-terminally extended nested sets of peptides identified in isolates from HLA-B*57:01. Peptides considered unlikely to be true HLA-B*57:01 ligands due to lack of conformation to consensus motif are noted. Alignment of sets is based on $\mathrm{P} 1$ of the minimal peptide except where the minimal peptide is 7 or 8 amino acids in length (in which case residue 1 is P3 or P2 respectively). Likely misaligns due to non-favourable amino acid at P2, and likely contaminants are noted. Maximal peptides used to describe the N-terminal extension motif are indicated. Peptides for which structures have been resolved and the register of the overhang observed are noted.

Supplementary Table 3: Extended sets containing C-terminal or $\mathrm{N}$ and C-terminal extensions. Peptides considered unlikely to be true HLA-B*57:01 ligands due to lack of conformation to consensus motif are noted. 


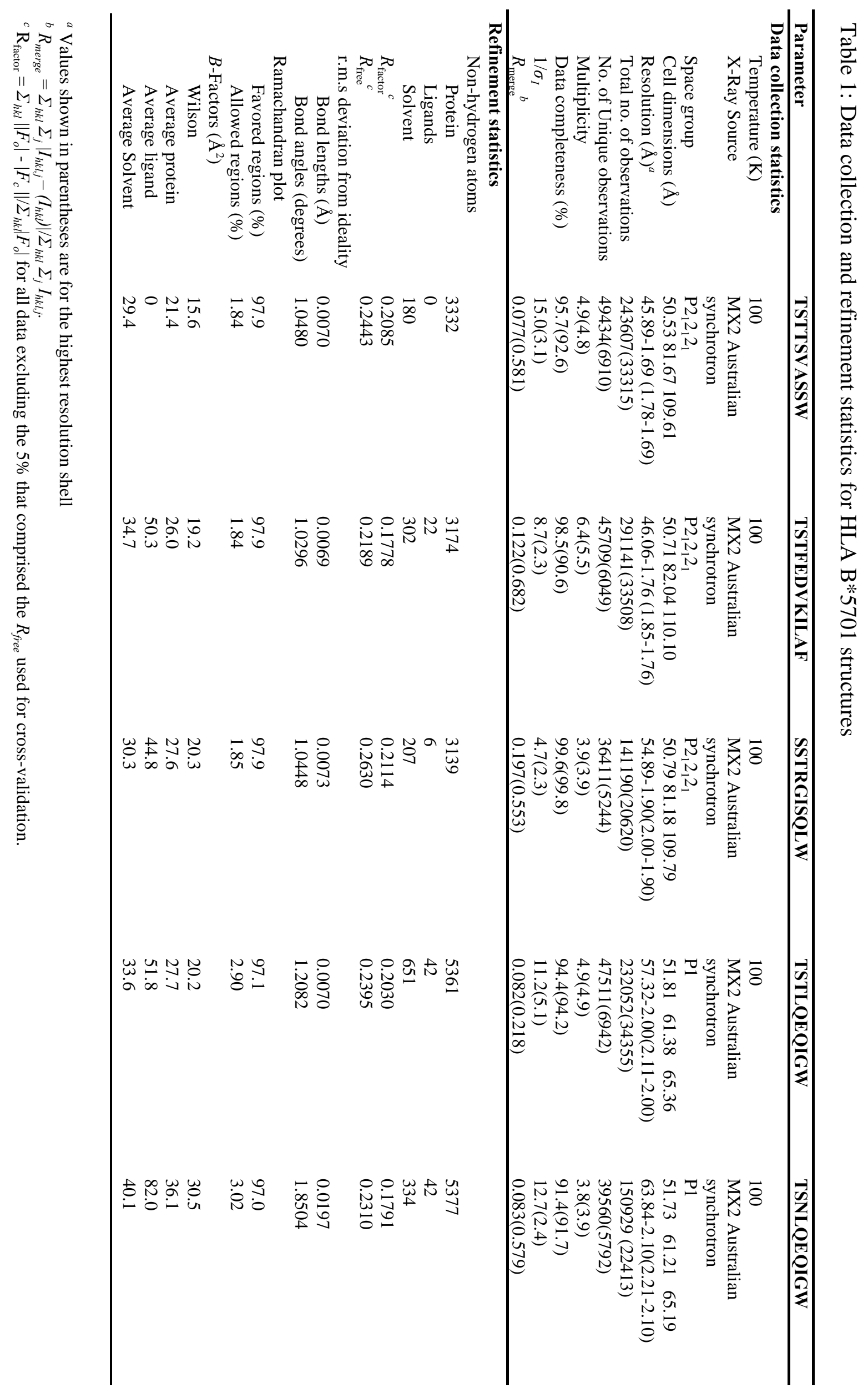


Figure 1

A.

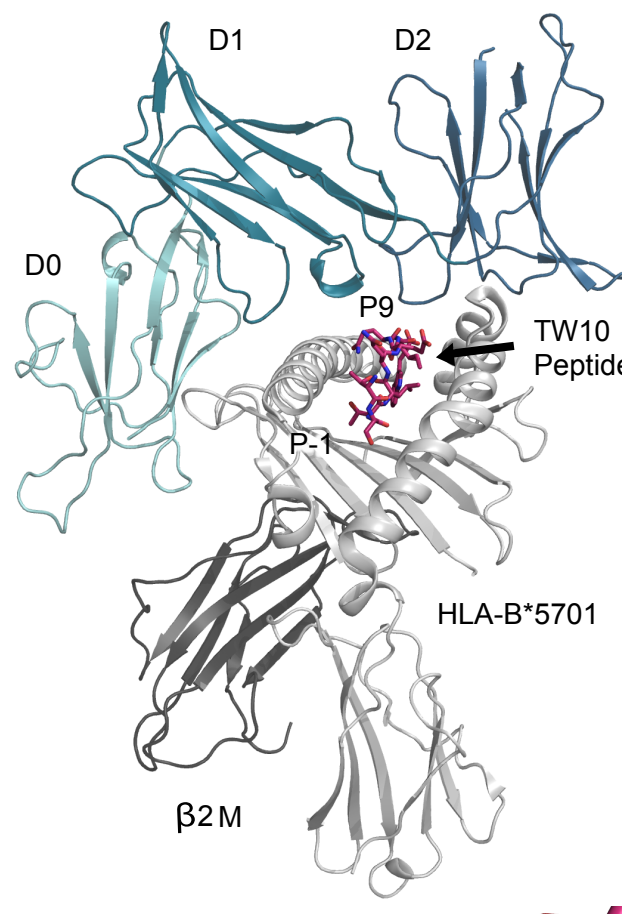

C.

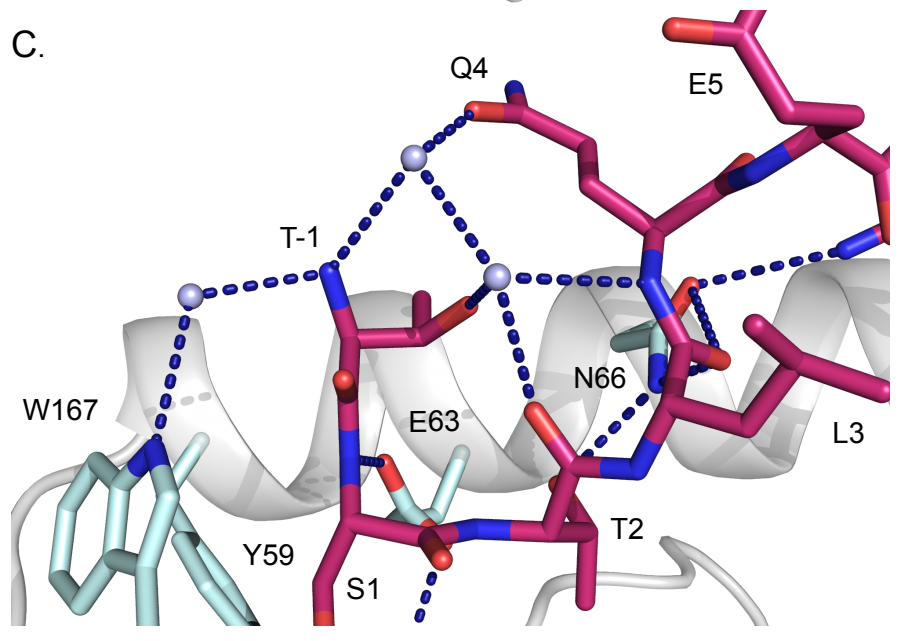

B.
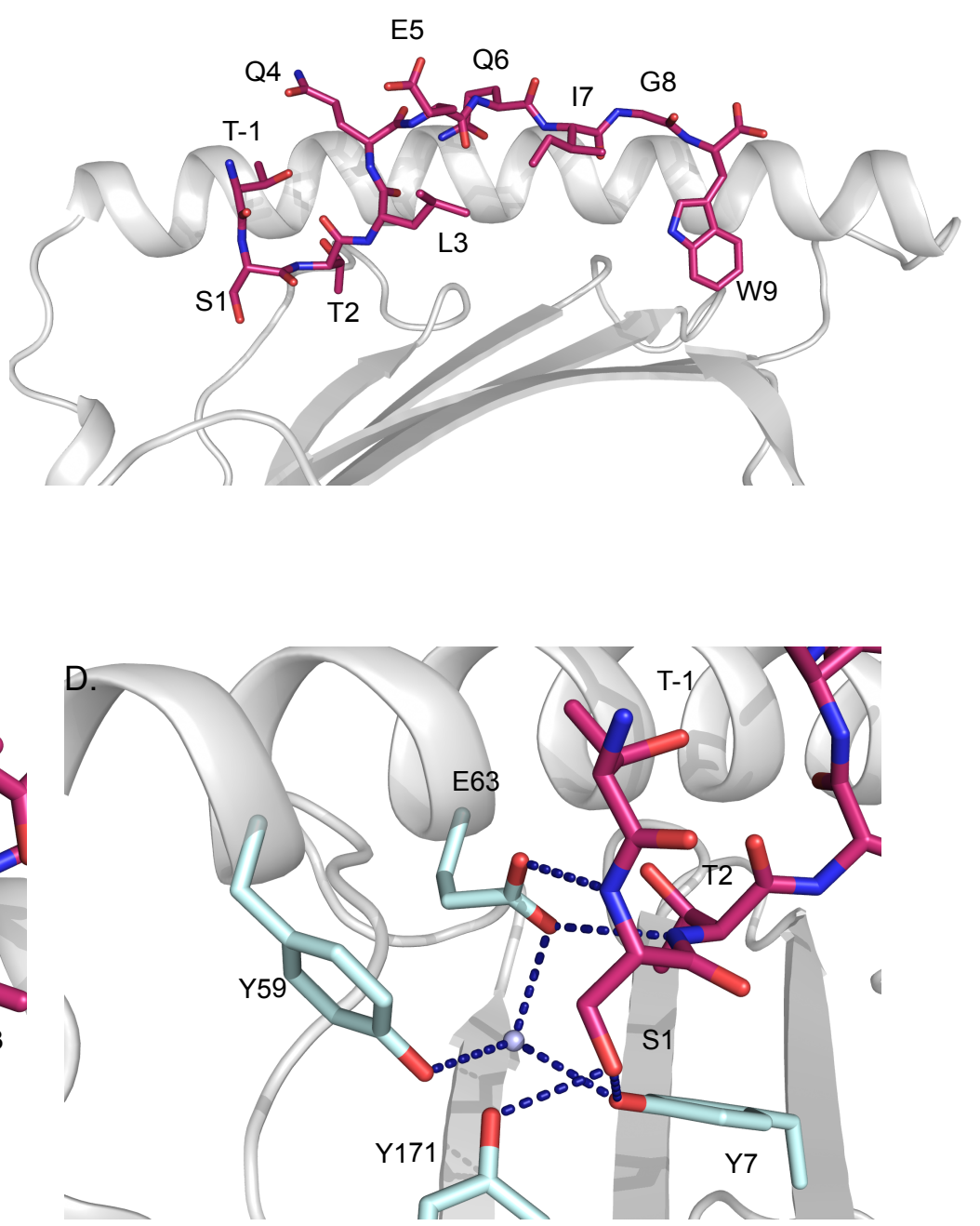
Figure 2

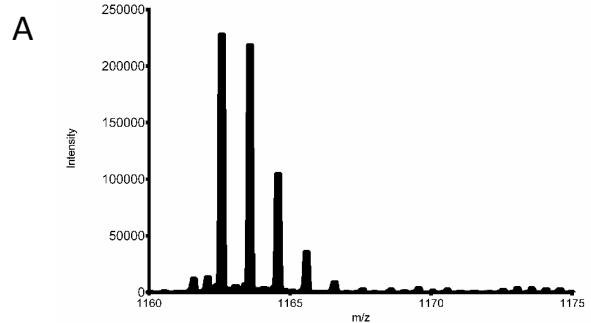

B

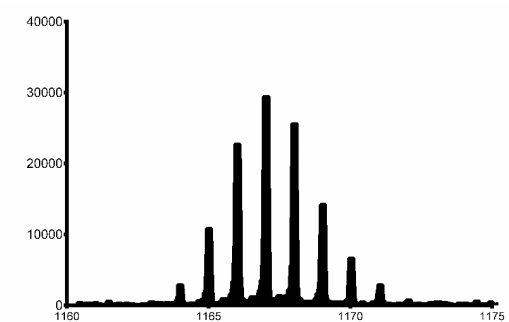

C

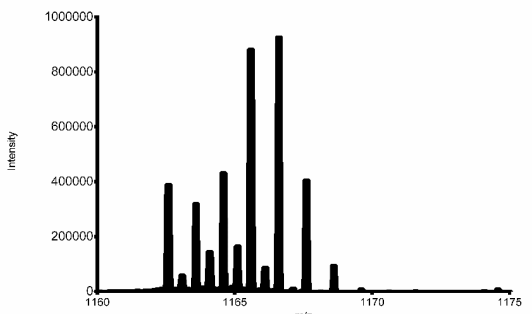


Figure 3

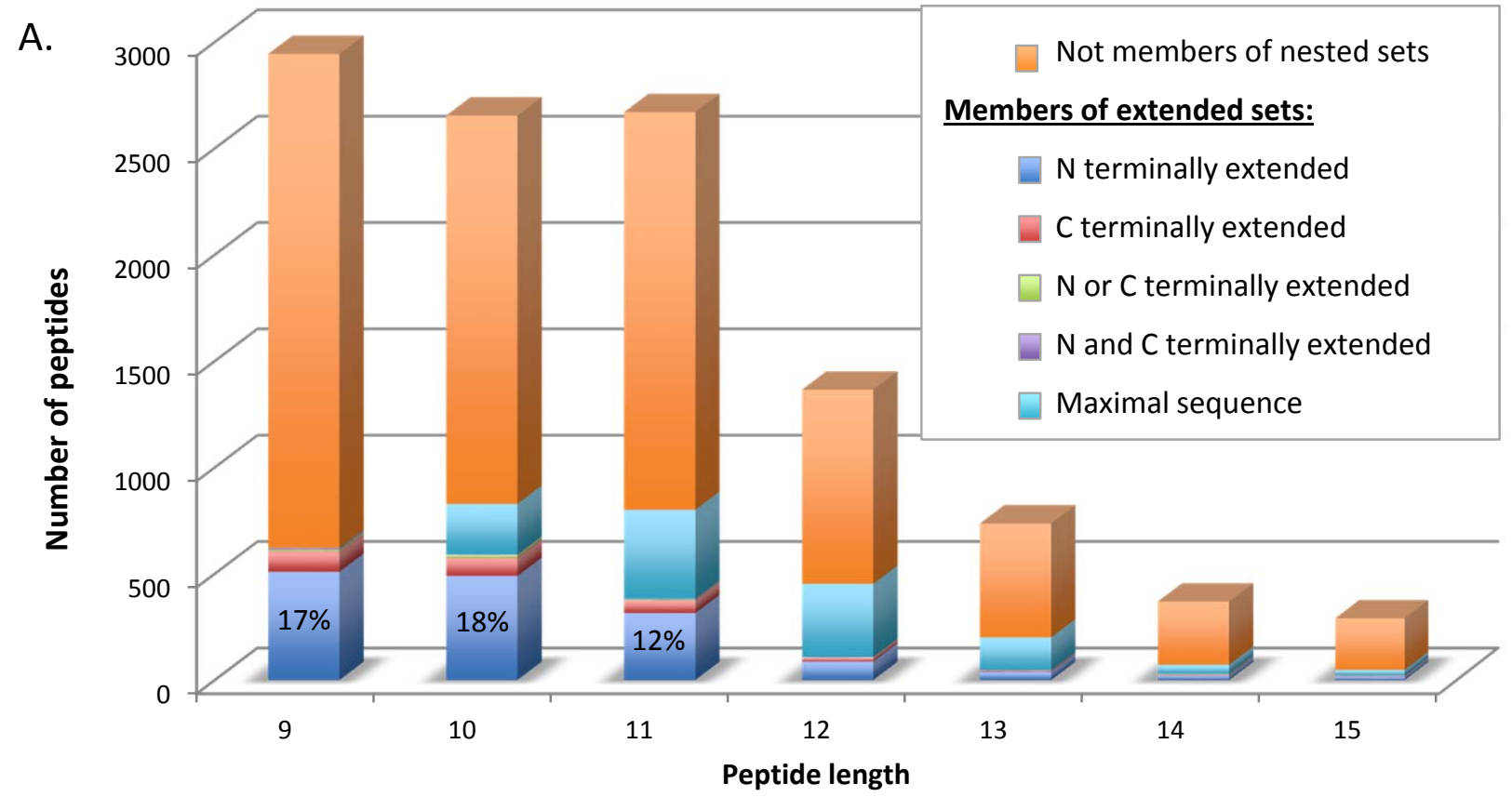

B. All 9 to 11 mer peptides

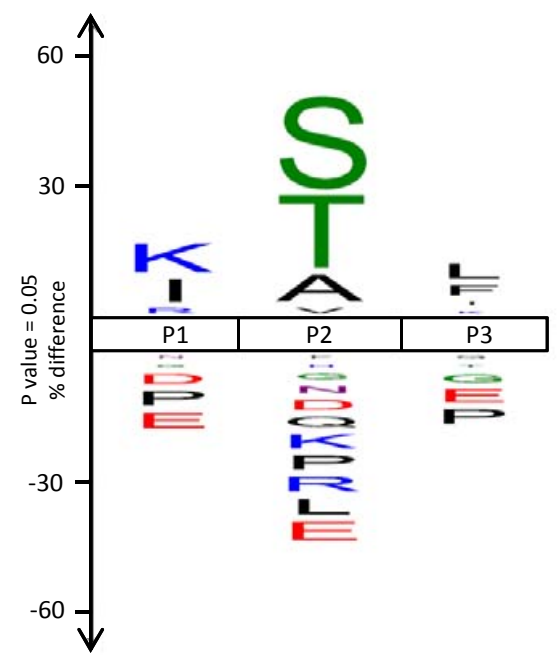

C. Maximal sequence of $\mathrm{N}$ terminally extended sets

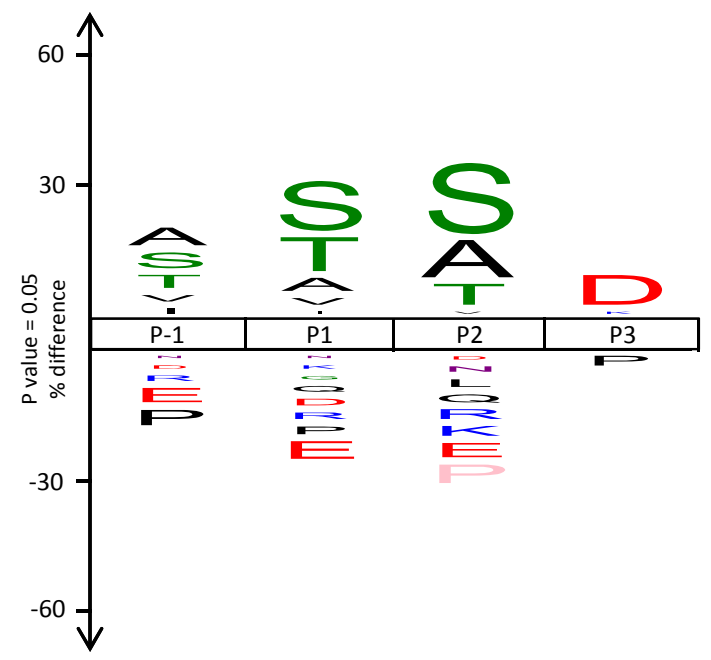


Figure 4

A.

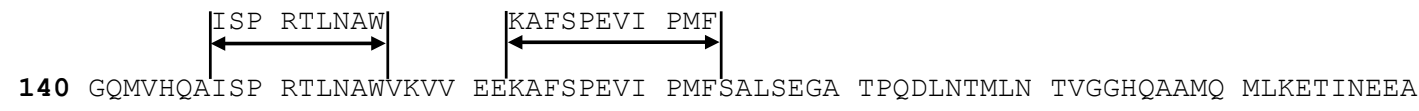

211 AEWDRVHPVH AGPIAPGQMR EPRGSDIAGT

281 TSILDIRQGP KEPFRDYVDR FYKTLRAEQA SQEVKNWMTE

B.

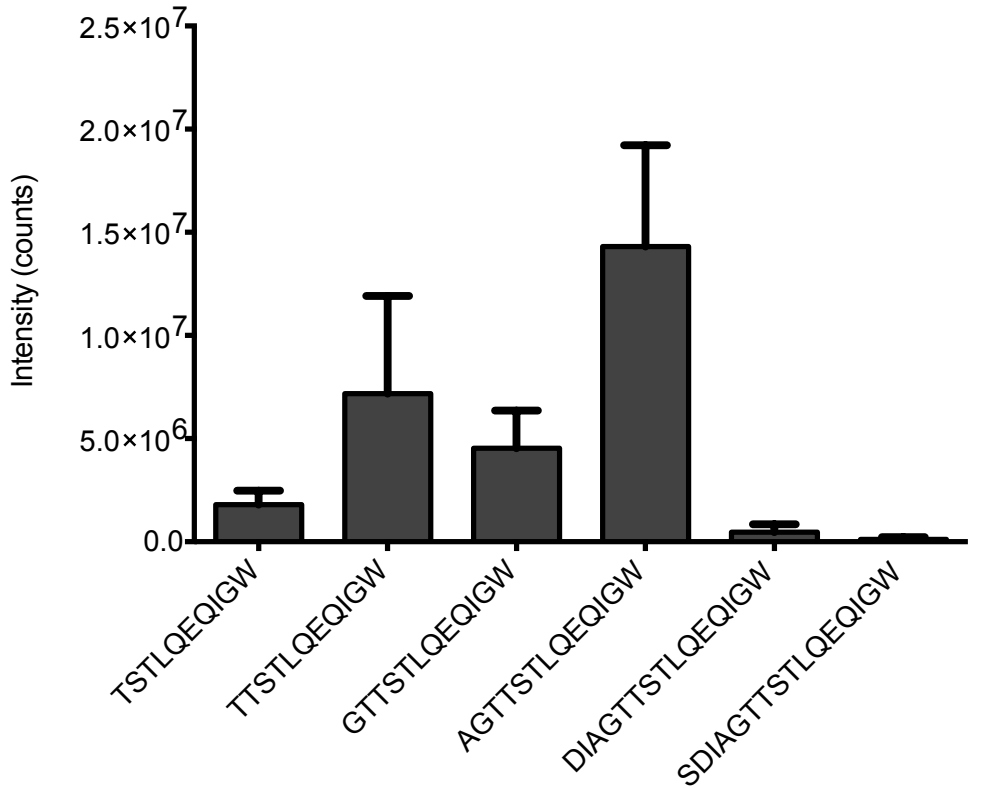


Figure 5

A.

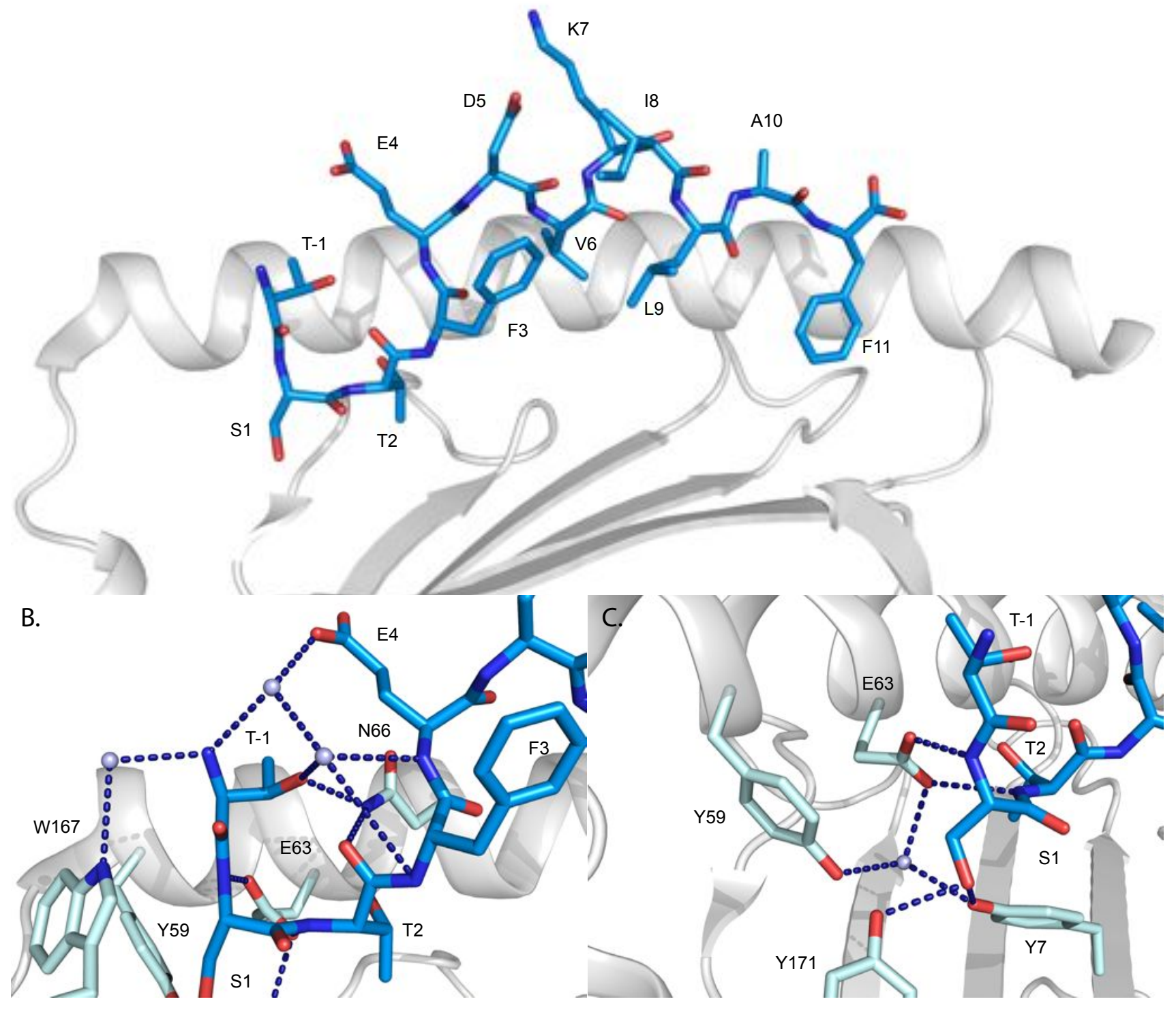


Figure 6

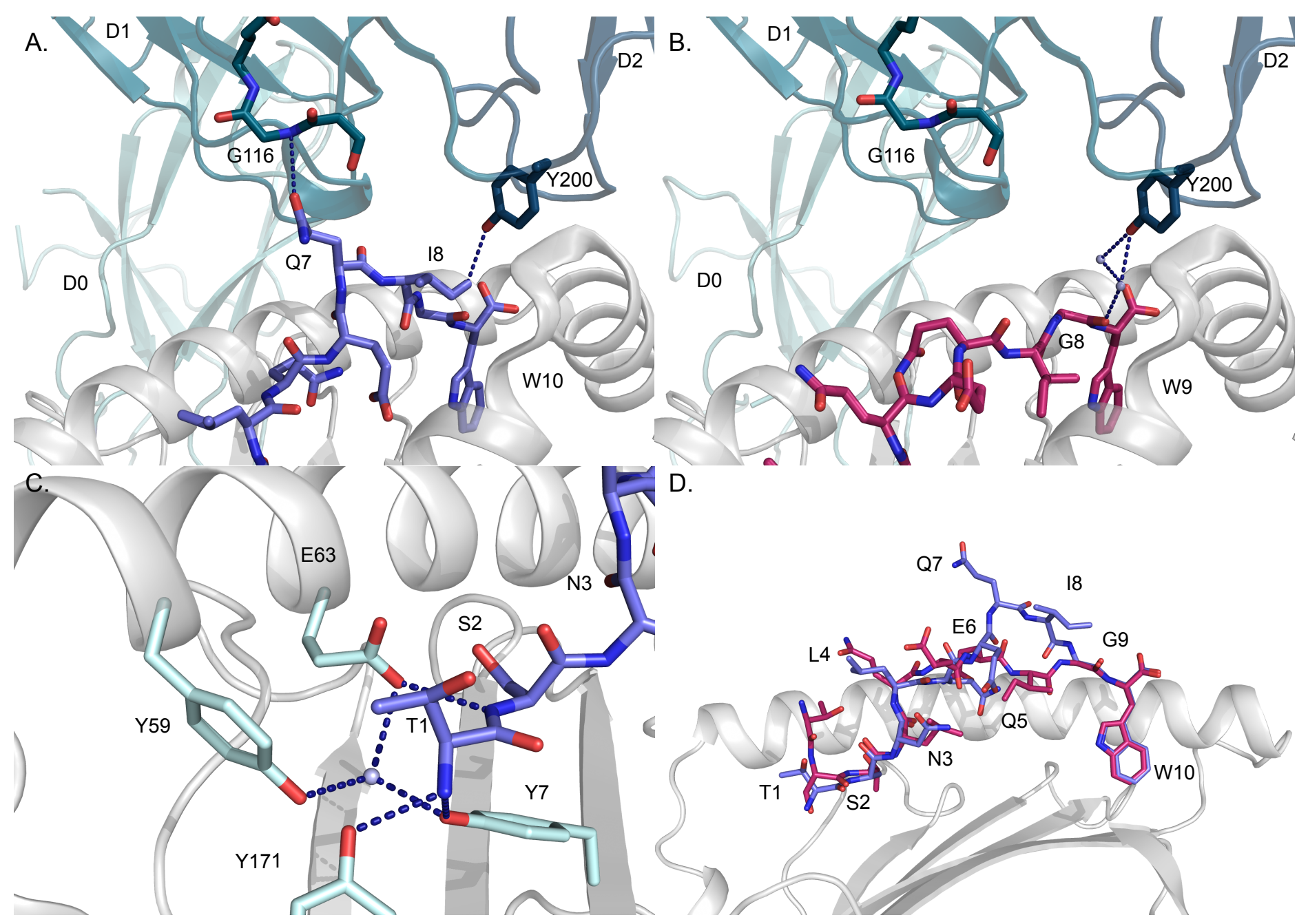


A.

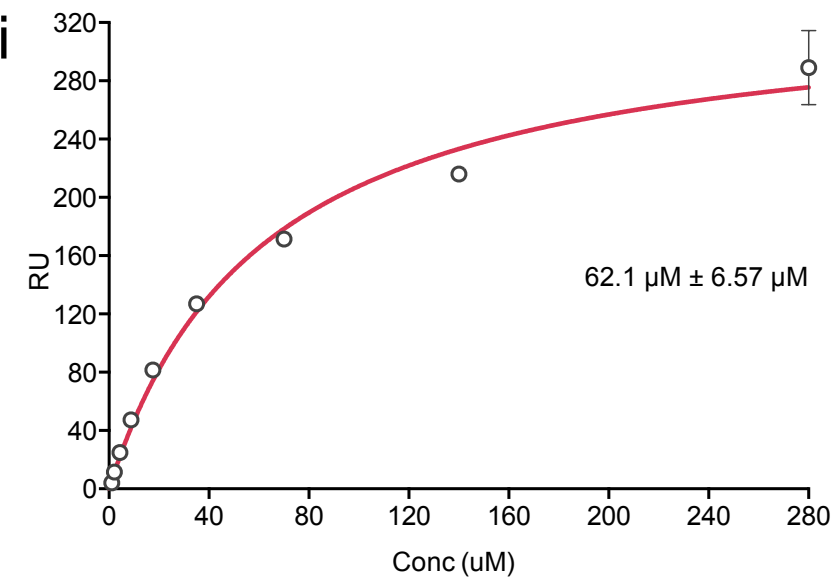

ii

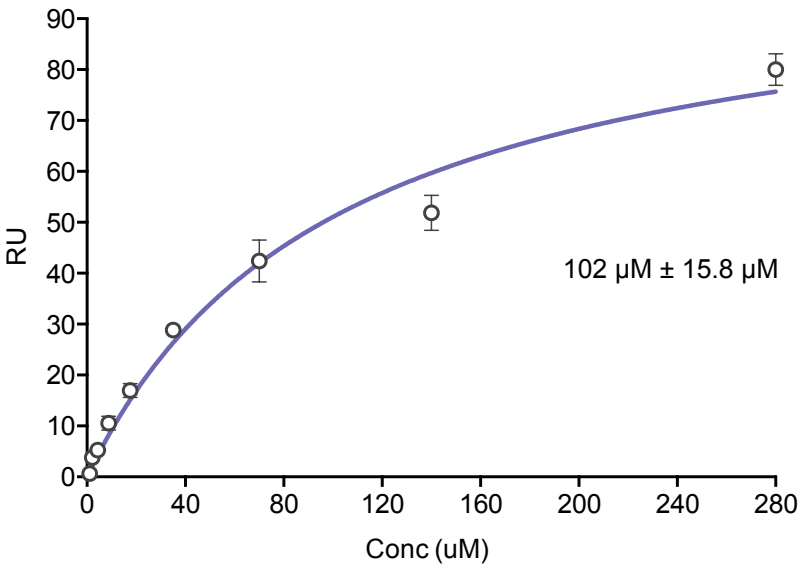

B.

Figure 7

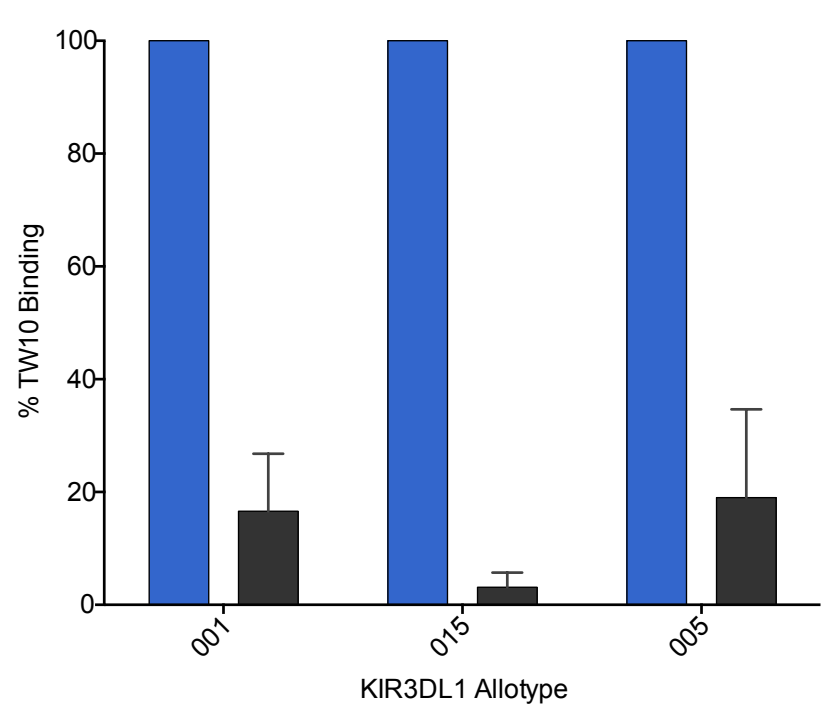

C.

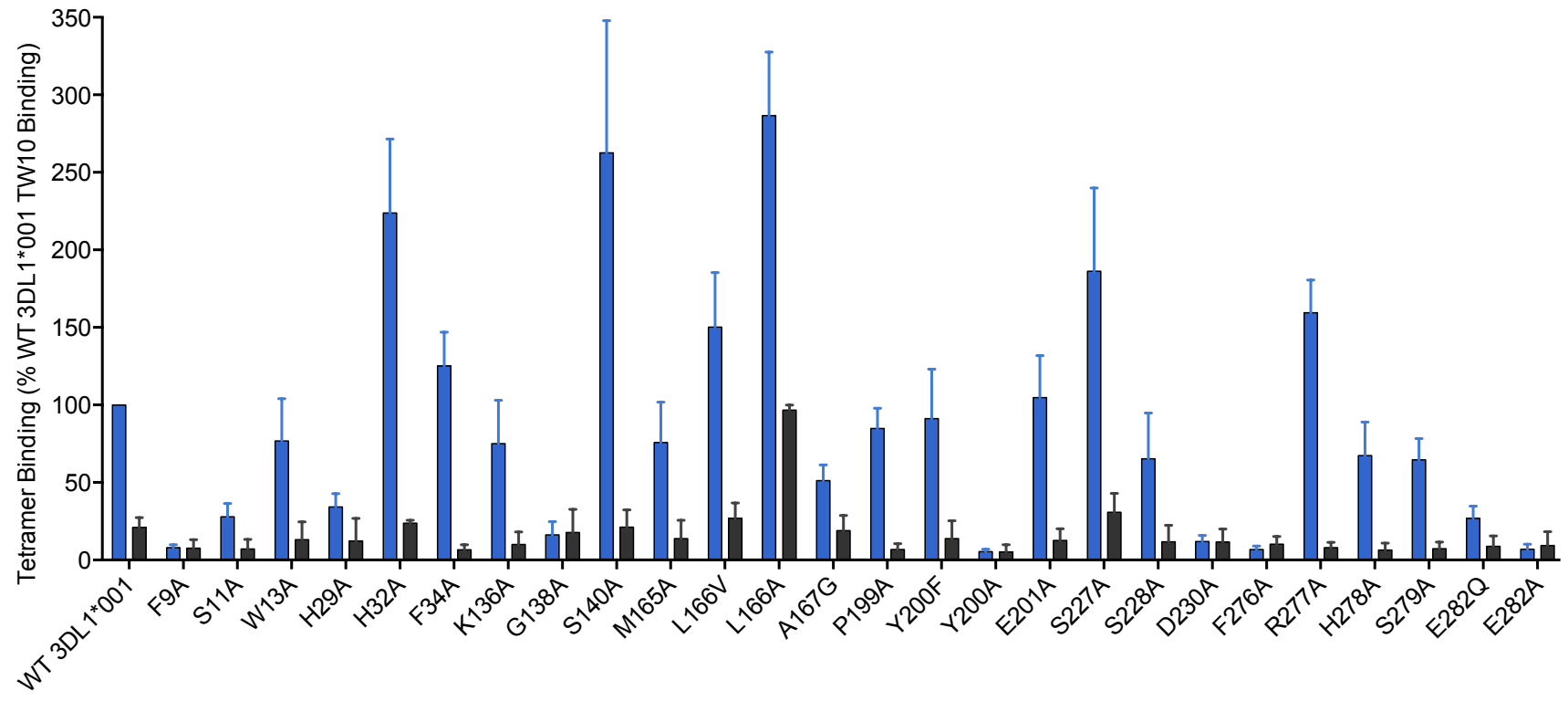

D.

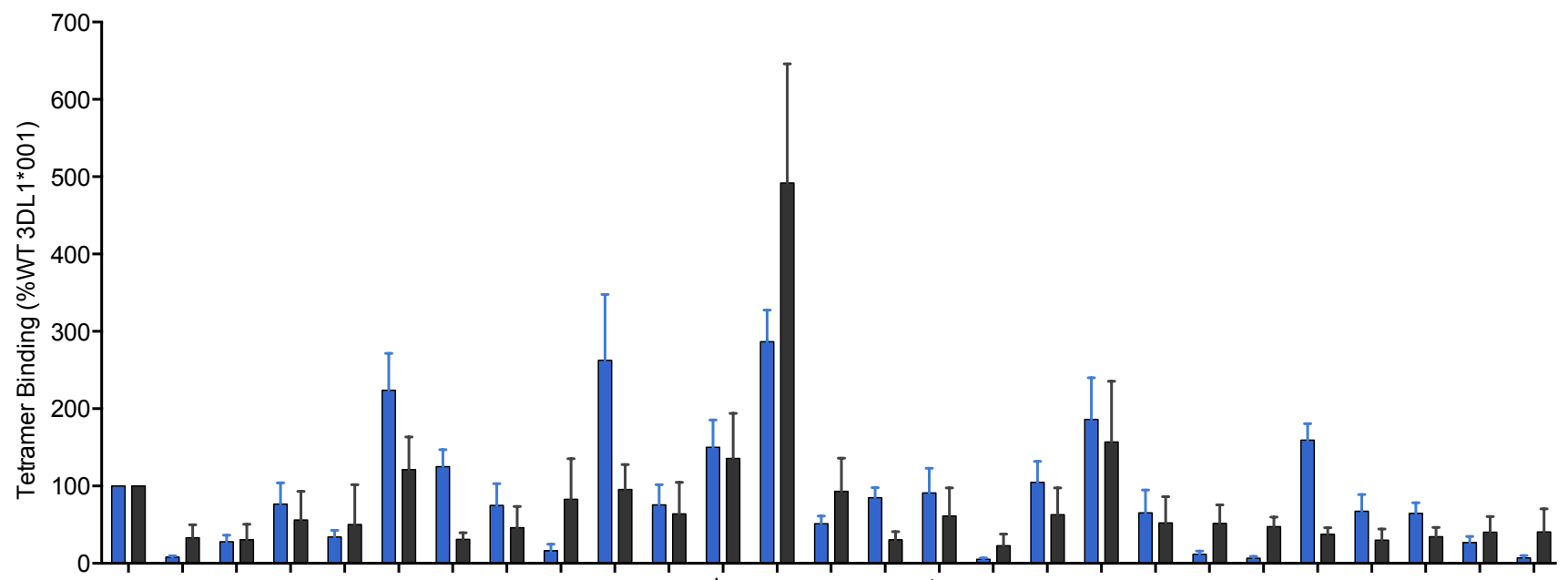

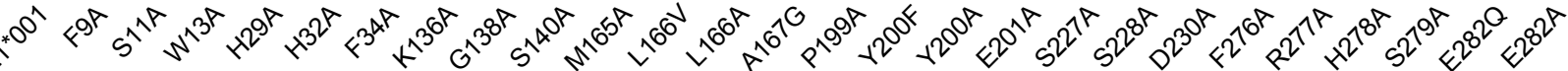


Supplementary Figure 1
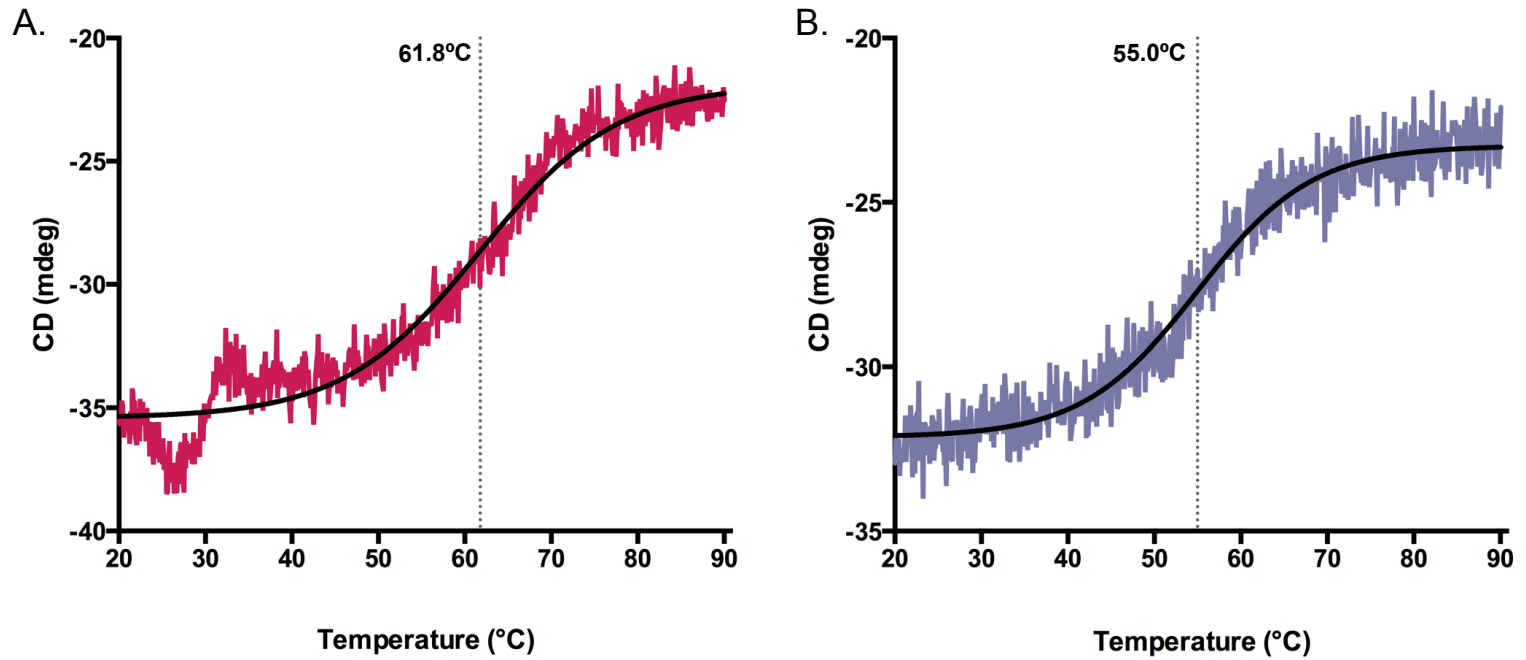